Interfaces and Free Boundaries 10 (2008), 301-338

\title{
The critical mass constraint in the Cahn-Hilliard equation
}

\author{
XIAOFENG REN ${ }^{\dagger}$ \\ Department of Mathematics, The George Washington University, Washington, DC 20052, USA \\ JUNCHENG WEI \\ Department of Mathematics, Chinese University of Hong Kong, Hong Kong, PRC
}

[Received 12 July 2006 and in revised form 28 January 2008]

\begin{abstract}
When the mass constraint of the Cahn-Hilliard equation in two dimensions is lowered to the order of $\epsilon^{2 / 3}$, where $\epsilon$ is the interface thickness parameter, the existence of droplet solutions becomes conditional. For interior single droplet solutions, there is a critical value for the mass constraint such that above this value two interior single droplet solutions exist, and below this value interior single droplet solutions cannot be constructed. One solution has smaller droplet radius than the other. The one with smaller radius is less stable. The center of the droplets in these solutions is (almost) the point in the domain that is farthest from the boundary. A critical mass constraint also appears when multiple droplet solutions are sought. Above the critical mass constraint, which now depends on the number of droplets, there exist two multi-droplet solutions. In each solution the radii of the droplets are about the same. However, when the two solutions are compared, one has larger droplet radius than the other. The locations of the droplets are determined by the solution of a disc packing problem.
\end{abstract}

\section{Introduction}

The Cahn-Hilliard equation was originally proposed to study binary alloys [9]. Let $u$ be the relative concentration of one of the two components in an alloy, so $1-u$ is the relative concentration of the other component. At a point $x$ where $u(x) \approx 1$ there is higher concentration of the first component, and at a point where $u(x) \approx 0$ there is high concentration of the second component. When $u(x)$ stays between 0 and 1, a mixture of the two components occupies $x$. Let $\Omega$ be the region taken by the alloy, which we assume to be a smooth and bounded domain. The average concentration of the first component is $|\Omega|^{-1} \int_{\Omega} u(x) \mathrm{d} x$, denoted by $m$, often called the mass constraint. Here $|\Omega|$ is the Lebesgue measure of $\Omega$.

In a dimensionless form the free energy of the system is

$$
I(u)=\int_{\Omega}\left(\frac{\epsilon^{2}}{2}|\nabla u|^{2}+F(u)\right) \mathrm{d} x .
$$

The function $F$ is smooth with at least quadratic growth rate at $\pm \infty$. It is a balanced double well potential with two global minimum points at 0 and 1 . Both minima are non-degenerate: $F^{\prime \prime}(0)>0$, $F^{\prime \prime}(1)>0$. Moreover, we assume that $F^{\prime \prime \prime}(0)<0$. There is a third critical point between 0 and 1 which is a local maximum. We impose a symmetry condition $F(u)=F(1-u)$. Then the local maximum point is $1 / 2$. The reader may take the particular example $F(u)=(1 / 4) u^{2}(1-u)^{2}$ throughout this paper.

\footnotetext{
Corresponding author. E-mail: ren@gwu.edu

E-mail: wei@ math.cuhk.edu.hk
} 
The functional $I$ is defined for $u$ in the admissible set

$$
\mathcal{A}=\left\{u \in W^{1,2}(\Omega): \frac{1}{|\Omega|} \int_{\Omega} u \mathrm{~d} x=m\right\}
$$

with $m \in(0,1)$, the mass constraint, being a given number. In this paper a bar over a function denotes its average. Hence $\bar{u}=m$.

The Euler-Lagrange equation derived from (1.1, 1.2$)$ is

$$
-\epsilon^{2} \Delta u+f(u)=\eta \quad \text { in } \Omega, \quad \partial_{\nu} u=0 \quad \text { on } \partial \Omega .
$$

The function $f$ is the derivative of $F$. If we use the particular $F(u)=(1 / 4) u^{2}(1-u)^{2}$, then $f(u)=u(u-1 / 2)(u-1) \cdot v$ is the outward normal direction on $\partial \Omega$ and $\partial_{v}$ is the directional derivative in that direction. Both the function $u$ and the constant $\eta$ are unknown in (1.3). The constant $\eta$ is the Lagrange multiplier coming from the constraint of $u$ in 1.2. If we integrate (1.3), then

$$
\eta=\overline{f(u)} \text {. }
$$

We introduce a nonlinear operator $S$ by

$$
S(u)=-\epsilon^{2} \Delta u+f(u)-\overline{f(u)},
$$

so the equation $(1.3)$ becomes

$$
S(u)=0 .
$$

To have low free energy the field $u(x)$ has to be close to 0 or 1 because this makes $F(u(x))$ small. Any oscillation between 0 and 1 makes $\left(\epsilon^{2} / 2\right)|\nabla u|^{2}$ large, and is best avoided. However, the constraint $\bar{u}=m \in(0,1)$ does not allow $u$ to be 0 (or 1 ) everywhere. The parameter $\epsilon$ is a small positive number. If $u(x)$ must vary between 0 and 1 , it can do so over a narrow region without raising the free energy too significantly.

Most works on this problem make the assumption that $m$ is independent of $\epsilon$. When $\Omega$ is a twodimensional domain, Alikakos and Fusco [3, 4], Alikakos, Bronsard and Fusco [1], and Alikakos, Fusco and Karali [5] studied the development of a bubble profile under a dynamical law of $I$. A bubble profile $u(x)$ is a function that is close to 1 inside a round circle, a bubble, of radius $r$ with $\pi r^{2} /|\Omega| \approx m$ and close to 0 outside the circle. There is a narrow transition region whose width is of order $\epsilon$ along the circle. In this region $u(x)$ changes rapidly from 1 to 0 . They showed that this profile is rather stable in the dynamics and the bubble moves slowly towards the nearest boundary point on $\partial \Omega$. One byproduct of their work is that there exists an equilibrium, which is a solution of [1.3, of the bubble profile. The location of the bubble in the equilibrium is not given in [4] or [1]. Wei and Winter [19] gave a static method, without using the dynamics of $I$, to show that a bubble equilibrium exists with the center of the bubble being the farthest point in $\Omega$ from $\partial \Omega$. A formal justification of the location of this bubble was given by Ward [18].

When $m$ is independent of $\epsilon$, one powerful technique to study the Cahn-Hilliard equation is $\Gamma$-convergence theory (cf. De Giorgi [11], Modica and Mortola [15], Modica [14], and Kohn and Sternberg [13]). It reduces the variational problem (1.1) to the geometric problem of the perimeter functional: Given a subset $E$ of $\Omega$ (again assume $\Omega \subset \mathbb{R}^{2}$ ) whose size is $m|\Omega|$, the perimeter functional $P_{\Omega}(E)$ associates to $E$ the arc length of the part of $\partial E$ that is in $\Omega$. One consequence of $\Gamma$-convergence theory is that as $\epsilon \rightarrow 0$, the global minimizer of $I$ must converge in some sense to 
a global minimizer of $P_{\Omega}$. For the global minimizer $E$ of $P_{\Omega}$, the part of the boundary of $E$ that is in $\Omega$ is a circular arc. $E$ also shares a part of its boundary with $\partial \Omega$. The arc meets $\partial \Omega$ at a right angle.

Another consequence of $\Gamma$-convergence theory is that if $E$ is an isolated local minimizer of $P_{\Omega}$, one can find a local minimizer $u$ of $I$ that is close to the characteristic function of $E$ if $\epsilon$ is sufficiently small. The set $\partial E \backslash \partial \Omega$ is approximated by the set $\{x: u(x)=1 / 2\}$. Using this fact Chen and Kowalczyk [10] proved that a small bubble solution exists if $m$ is sufficiently small. The bubble is attached to the boundary $\partial \Omega$ at a point whose mean curvature attains a local maximum, viewed from inside $\Omega$. Even though $m$ is a small number, it must be independent of $\epsilon$ in the $\Gamma$-convergence framework. On the other hand, Sternberg and Zumbrun [17] showed that in a strictly convex domain the interface must be connected.

Alikakos, Chen and Fusco [2] studied the dynamics of a boundary bubble profile using another dynamical law of $I$. Allowing $m$ to depend on $\epsilon$, they made an interesting discovery: To observe boundary bubble dynamics and to have the existence of boundary bubble equilibrium, the mass constraint cannot be too small, in terms of $\epsilon$. It was shown that the mass constraint $m$ can be of order $\epsilon^{2 / 3}$ at the lowest. They called the boundary bubble profile in the case $m \sim \epsilon^{2 / 3}$ the droplet profile. If one writes $m=\epsilon^{2 / 3} m_{0}+o\left(\epsilon^{2 / 3}\right)$, a critical value for $m_{0}$ exists. Below this value one cannot construct a good approximate solution meeting all the requirements in their droplet dynamics analysis. This droplet profile has its root in the bubble profile when $m$ is independent of $\epsilon$. When we decrease $m$ to $\epsilon^{2 / 3}$ order, the bubble shrinks to a droplet.

If the mass constraint is above the critical level but still of order $\epsilon^{2 / 3}$, it was shown in [2] that there is a second solution with a boundary droplet. This droplet has smaller radius than the first one. It is less stable and has higher free energy. The existence of this second solution has its root in the so called spike solutions.

When $m$ is not too close to $1 / 2$ but independent of $\epsilon$, one can find a solution that is close to $m$ for most $x \in \Omega$, except in a neighborhood of a point where the graph of the solution has a sharp peak. This point may be on $\partial \Omega$ or inside of $\Omega$. The solution is very unstable and has high free energy. For more information about spike solutions in this parameter range see Bates and Fife [7], Bates, Dancer and Shi [6], Bates and Fusco [8], and Wei and Winter [20, 21]. When $m$ is decreased to the $\epsilon^{2 / 3}$ range, a boundary spike solution flattens to become a boundary droplet solution. This droplet solution is different from the earlier one. It has smaller radius and is less stable.

In this paper we study interior droplet solutions under the mass constraint $m \sim \epsilon^{2 / 3}$ in two dimensions: $\Omega \subset \mathbb{R}^{2}$. More explicitly, we assume

$$
m=\epsilon^{2 / 3} m_{0}+o\left(\epsilon^{2 / 3}\right)
$$

with $m_{0}>0$ independent of $\epsilon$. In the case of $\Omega$ being a unit disc, interior single droplet solutions may be studied within the class of radially symmetric functions. In this class it was shown in [2] that a critical mass constraint exists. When the mass constraint is in the $\epsilon^{2 / 3}$ range and above the critical level, the droplet solutions and the constant solution have comparable free energy of order $\epsilon^{4 / 3}$. We will show in this paper that a critical mass constraint also exists in the general domain $\Omega$ for interior single droplet solutions. If the mass constraint is above the critical level and still of order $\epsilon^{2 / 3}$ we find two interior droplet solutions. One of them has greater radius and is related to an interior bubble solution (see [19]). The second one has smaller radius and is related to an interior spike solution (see [21]). Both solutions are unstable. Of the two solutions, the one with smaller droplet is less stable. 
Our approach is static. We do not use any of the dynamic laws associated with $I$. We use a type of Lyapunov-Schmidt reduction procedure tailored for singularly perturbed problems. To understand this method we must have a good understanding of the linearized operator at the solution we want to construct. The linear operator admits eigenvalues that tend to 0 as $\epsilon \rightarrow 0$, which we call critical eigenvalues. These eigenvalues are further divided according to the rates at which they converge to 0 . They give us a split into a finite-dimensional manifold $\mathcal{M}$, and at each point, say $w_{\xi}$, of the manifold, an infinite-dimensional fiber space $\mathcal{F}_{\xi}$. In this construction every member in $\mathcal{M}$ is a function with a droplet profile. The center of the droplet is at $\xi$ which serves to parametrize $\mathcal{M}$. In each fiber space we look for a function $\phi_{\xi} \in \mathcal{F}_{\xi}$ so that $w_{\xi}+\phi_{\xi}$ "solves" the equation (1.3) in the fiber direction. Now $w_{\xi}+\phi_{\xi}$ forms another manifold, say $\mathcal{N}$. We maximize $I$ in $\mathcal{N}$. The maximum is achieved at a particular $\xi$ which we call $\xi_{*}$. Then $w_{\xi_{*}}+\phi_{\xi_{*}}$ is an exact solution of the equation (1.3). This approach has been used to study the Cahn-Hilliard problem by Wei and Winter in [19, 20, 21].

It turns out that maximizing $I\left(w_{\xi}+\phi_{\xi}\right)$ with respect to $\xi$ is equivalent to maximizing the distance of $\xi$ to the boundary of $\Omega$. Therefore this approach also gives us the location of the droplet in a solution. The center $\xi_{*}$ of the droplet is (almost) the point in $\Omega$ that is farthest from $\partial \Omega$.

We will also show the existence of solutions with multiple droplets. Here given any positive integer $K$, we find a critical mass constraint and, above this critical level, two solutions, each of which has a profile of $K$ droplets. In each solution the droplets are almost of the same size. However, if we compare the two $K$-droplet solutions, one solution has smaller droplets than the other.

The locations of the droplets in both solutions are determined by solving a disc packing problem. In the disc packing problem we are given $K$ (open) discs of the same radius. What is the greatest possible radius of these discs so that they can all be placed inside $\Omega$ without intersection? Let $\xi^{1}, \ldots, \xi^{K}$ be the centers of $K$ discs. If we take the radius of the discs to be

$$
\varphi\left(\xi^{1}, \ldots, \xi^{K}\right)=\min \left\{d_{\xi^{k}},\left|\xi^{l}-\xi^{m}\right| / 2: k, l, m \in\{1, \ldots, K\}, l \neq m\right\},
$$

where $d_{\xi^{k}}$ is the distance of $\xi^{k}$ to $\partial \Omega$,

$$
d_{\xi^{k}}=\min \left\{\left|x-\xi^{k}\right|: x \in \partial \Omega\right\},
$$

then the discs are all inside $\Omega$ and they are mutually disjoint. To find the greatest possible radius, we simply maximize $\varphi\left(\xi^{1}, \ldots, \xi^{K}\right)$. The locations of the droplets of our multi-droplet solutions are (almost) the $\xi^{1}, \ldots, \xi^{K}$ that maximize $\varphi$.

The paper is organized as follows. In Section 2 we describe the shape of a droplet. In Section 3 we show the existence of two radially symmetric single droplet solutions in the unit disc, using a straightforward fixed point argument. In Section 4 we analyze the linear operator at each of the two radial solutions. We obtain detailed information on the eigenvalues of the linear operator. Equipped with this information we construct two interior droplet solutions in a general domain using the Lyapunov-Schmidt reduction method in Section 5. Finally, in Section 6 we find two solutions of multiple interior droplets. To do so, we employ the Lyapunov-Schmidt method to reduce the problem to the disc packing problem. Some of the proofs are quite technical. To help the reader follow the main framework of this paper, we leave these difficult proofs to the appendices.

To avoid overly complicated notations, a quantity's dependence on $\epsilon$ is usually suppressed. For instance we write $I$ instead of $I_{\epsilon}$ and $S$ instead of $S_{\epsilon}$. On the other hand, if a quantity is independent of $\epsilon$, we often use a subscript ${ }_{0}$ to emphasize this fact, such as $m_{0}$ in $m=\epsilon^{2 / 3} m_{0}+o\left(\epsilon^{2 / 3}\right)$. We use $C, C_{0}, C_{1}, \ldots$ and $a, a_{0}, a_{1}, \ldots$, to denote positive constants independent of $\epsilon$. Their values change from line to line and even from place to place in the same line. 


\section{The droplet profile}

The shape of a droplet is described by the solution of the equation

$$
-\epsilon^{2} \Delta v+f(v)=\beta
$$

in the entire space $\mathbb{R}^{2}$. This solution $v$ is radially symmetric. In the language of the formal asymptotic theory, $v$ is known as an inner approximation. Also note that the two-parameter problem (2.1) may be reduced to the one-parameter, $\beta$, problem by scaling the input variable of $v$, hence eliminating $\epsilon^{2}$. We collect some well-known results about $v$ in this section.

The constant $\beta$ on the right side is assumed to be positive and have the expansion

$$
\beta=\epsilon^{2 / 3} \beta_{0}+o\left(\epsilon^{2 / 3}\right)
$$

with $\beta_{0}>0$ independent of $\epsilon$. Denote the three zeros of $f-\beta$ by $z, z^{\prime}, z^{\prime \prime}$, in increasing order. Here $z$ is positive and $z^{\prime \prime}$ is greater than 1 . Because $\beta \sim \epsilon^{2 / 3}$,

$$
z \sim \epsilon^{2 / 3}, \quad z^{\prime \prime}-1 \sim \epsilon^{2 / 3} .
$$

The interface of the droplet profile is identified by $\rho>0$ where

$$
v(\rho)=1 / 2 .
$$

So for $r>\rho$, when $\epsilon$ is small $v(r)$ is close to $z$, and for $r<\rho, v(r)$ is close to $z^{\prime \prime}$. It is known that $\rho \sim \epsilon^{1 / 3}$ (see for example [19, Lemma 2.1]). We therefore write

$$
\rho=\epsilon^{1 / 3} \rho_{0}+o\left(\epsilon^{1 / 3}\right) .
$$

$v$ decays to $z$ as $r=|x| \rightarrow \infty$ :

$$
\lim _{r \rightarrow \infty} v(r)=z
$$

The decay rates of $v$ and $v^{\prime}$ are given as follows (see [19, Lemma 2.8]).

LEMma 2.1 There exist positive constants $C_{0}, C_{1}, a_{0}, a_{1}$ independent of $\epsilon$ such that

$$
C_{0} e^{-a_{0} \epsilon^{-2 / 3}} e^{-\sqrt{f^{\prime}(z)} r / \epsilon} \leqslant v(r)-z, \quad-v^{\prime}(r) \leqslant C_{1} e^{a_{1} \epsilon^{-2 / 3}} e^{-\sqrt{f^{\prime}(z) r} / \epsilon} .
$$

Near $\rho$ we have the following expansion formula.

Lemma 2.2 Near $\rho, v$ can be expanded as

$$
v(\epsilon t+\rho)=H(t)+\epsilon^{2 / 3} P(t)+\epsilon^{4 / 3} Q(t)+o\left(\epsilon^{4 / 3}\right)
$$

where $H, P$ and $Q$ are respectively the solutions of

$$
\begin{gathered}
-H^{\prime \prime}+f(H)=0, \quad H(-\infty)=1, H(\infty)=0, H(0)=1 / 2 ; \\
-P^{\prime \prime}+f^{\prime}(H) P=\frac{\epsilon^{1 / 3}}{\rho} H^{\prime}+\text { const }, \quad P(0)=0 ; \\
-Q^{\prime \prime}+f^{\prime}(H) Q=\frac{\epsilon^{1 / 3}}{\rho} P^{\prime}-\frac{\epsilon^{2 / 3}}{\rho^{2}} t H^{\prime}-\frac{1}{2} f^{\prime \prime}(H) P^{2}, \quad Q(0)=0 .
\end{gathered}
$$


For the proof we refer the reader to [16, Section 2], particularly [16, Lemma 2.3]. There we studied the more complex Ohta-Kawasaki model of diblock copolymers which in addition to the two terms in 1.1 has a nonlocal term. The reader can simply ignore that nonlocal term when applying the results there.

In (2.4), const is a constant determined by the solvability condition

$$
\int_{\mathbb{R}}\left(\frac{\epsilon^{1 / 3}}{\rho} H^{\prime}+\text { const }\right) H^{\prime} \mathrm{d} t=0
$$

If we relate const to $\beta$, we find the following important relation between $\beta_{0}$ and $\rho_{0}$.

LEMMA 2.3 In the expansion $\rho=\epsilon^{1 / 3} \rho_{0}+o\left(\epsilon^{1 / 3}\right), \rho_{0}$ satisfies $\beta_{0}=\tau / \rho_{0}$ where $\tau$ is a constant given by $\tau=\int_{\mathbb{R}}\left(H^{\prime}(t)\right)^{2} \mathrm{~d} t$.

The constant $\tau$ is independent of $\epsilon$. It can also be given by

$$
\tau=\int_{0}^{1} \sqrt{2 F(q)} \mathrm{d} q .
$$

These two definitions are equivalent because of the first integral $-\frac{1}{2}\left(H^{\prime}\right)^{2}+F(H)=0$ of $H$. Note that $H^{\prime}=\sqrt{2 F(H)}$ and hence

$$
\int_{\mathbb{R}}\left(H^{\prime}(t)\right)^{2} \mathrm{~d} t=\int_{\mathbb{R}} \sqrt{2 F(H(t))} H^{\prime}(t) \mathrm{d} t=\int_{0}^{1} \sqrt{2 F(H)} \mathrm{d} H .
$$

$\tau$ is known as the surface tension.

Proof of Lemma 2.3. From 2.6 we find

$$
\text { const }=\frac{\epsilon^{1 / 3}}{\rho} \int_{\mathbb{R}}\left(H^{\prime}\right)^{2} \mathrm{~d} t=\frac{\epsilon^{1 / 3} \tau}{\rho} .
$$

If we send $\epsilon \rightarrow 0$, then const $=\epsilon^{1 / 3} \tau / \rho \rightarrow \tau / \rho_{0}$. On the other hand, by 2.2 and Lemma 2.2, $z=v(\infty)=\epsilon^{2 / 3} P(\infty)+o\left(\epsilon^{2 / 3}\right)$ and, by (2.4) of Lemma 2.2, $f^{\prime}(0) P(\infty)=$ const. Hence $z_{0} f^{\prime}(0)=\mathrm{const}+o(1)$. But $\beta_{0}=f^{\prime}(0) z_{0}$. Therefore const $\rightarrow \beta_{0}$ as $\epsilon \rightarrow 0$. Hence $\beta_{0}=\tau / \rho_{0}$.

\section{The radial case}

We take $\Omega$ to be the unit disc:

$$
\Omega=\left\{x \in \mathbb{R}^{2}:|x|<1\right\} .
$$

All functions that appear in this section are radially symmetric. We prove the following theorem.

THEOREM 3.1 Let $\Omega$ be a unit disc. If the mass constraint $m$ is chosen so that $m_{0}>$ $3\left(\tau /\left(2 f^{\prime}(0)\right)\right)^{2 / 3}$, then there exist two droplet solutions in $\Omega$.

The proof of the theorem consists of two steps. First we construct two approximate solutions that satisfy the Neumann boundary condition, the mass constraint, and up to an exponentially small error almost satisfy the differential equation. In the second step we use each of the two approximate solutions and find an exact solution nearby, using a fixed point argument. To this end we analyze the linearized operator. Most importantly, we show that the linearized operator is invertible and the spectrum is bounded away from 0 by a distance of order $\epsilon^{4 / 3}$. 
An approximate solution takes the form

$$
w(x)=v(x)+g(x)
$$

where $v$ is the radial droplet profile given in Section 2. The function $g(x)$ is the radial solution of the linear equation

$$
-\epsilon^{2} \Delta g+f^{\prime}(z) g=0 \quad \text { in } \Omega, \quad \partial_{\nu} g=-\partial_{\nu} v \quad \text { on } \partial \Omega .
$$

This correction function $g$ is quite small. We denote the $L^{\infty}(\Omega)$ norm of a function by $\|\cdot\|_{\infty}$ in this section.

Lemma $3.2\|g\|_{\infty}=O\left(\epsilon e^{-\sqrt{f^{\prime}(z)} / \epsilon}\right)$. Moreover, for any small $\iota>0$,

$$
g(r)=O\left(e^{-\left(\sqrt{f^{\prime}(z)}+\delta_{1}\right) / \epsilon}\right) \quad \text { if } r \leqslant 1-\iota
$$

for some $\delta_{1}>0$.

Proof. We write $g(r)=-v^{\prime}(1) \hat{g}(r)$. Then $v^{\prime}(1)=O\left(e^{-\sqrt{f^{\prime}(z)} / \epsilon}\right)$. The function $\hat{g}$ satisfies the equation

$$
-\epsilon^{2} \Delta \hat{g}+f^{\prime}(z) \hat{g}=0, \quad \partial_{\nu} \hat{g}=1 \quad \text { on } \partial \Omega .
$$

For a small $\iota>0, \hat{g}(r)=O(\epsilon)$ if $r>1-\iota$. If $r \leqslant 1-\iota$, there is $\delta_{1}>0$ so that $\hat{g}(r)=O\left(e^{-\delta_{1} / \epsilon}\right)$. More details of this proof may be found in [19].

The construction of $g$ ensures that $w$ satisfies the Neumann boundary condition. By adjusting $\beta$, or equivalently $\rho$ or $z$, we will make $w$ satisfy the mass constraint

$$
\bar{w}=m .
$$

The following is required by 3.1.

LEMMA 3.3 The constants $\rho_{0}$ and $m_{0}$ must satisfy the equation

$$
\rho_{0}^{2}+\frac{\tau}{\rho_{0} f^{\prime}(0)}=m_{0} .
$$

So $m_{0}$ cannot be less than $3\left(\tau /\left(2 f^{\prime}(0)\right)\right)^{2 / 3}$. This last value for $m_{0}$ is attained if $\rho_{0}=$ $\left(\tau /\left(2 f^{\prime}(0)\right)\right)^{1 / 3}$.

Proof. Because $g$ is exponentially small, $\bar{w}$ is exponentially close to $\bar{v}$. The integral of $v$ inside the interface $\rho$ is

$$
\pi \rho^{2}+o\left(\epsilon^{2 / 3}\right)=\pi \epsilon^{2 / 3} \rho_{0}^{2}+o\left(\epsilon^{2 / 3}\right),
$$

and the integral of $v$ outside the interface is

$$
\pi z+o\left(\epsilon^{2 / 3}\right)=\frac{\pi \beta}{f^{\prime}(0)}+o\left(\epsilon^{2 / 3}\right)=\frac{\pi \epsilon^{2 / 3} \tau}{\rho_{0} f^{\prime}(0)}+o\left(\epsilon^{2 / 3}\right)
$$

as $\beta=f(z)=f^{\prime}(0) z+o(z)$ and by Lemma 2.3 The mass constraint implies that

$$
\pi m=\pi \epsilon^{2 / 3} \rho_{0}^{2}+\frac{\pi \epsilon^{2 / 3} \tau}{\rho_{0} f^{\prime}(0)}+o\left(\epsilon^{2 / 3}\right) .
$$


This gives the relation

$$
m_{0}=\rho_{0}^{2}+\frac{\tau}{\rho_{0} f^{\prime}(0)} .
$$

For this equation to have a solution for $\rho_{0}, m_{0}$ cannot be too small. The smallest value for $m_{0}$ is $3\left(\tau /\left(2 f^{\prime}(0)\right)\right)^{2 / 3}$, which is attained if $\rho_{0}=\left(\tau /\left(2 f^{\prime}(0)\right)\right)^{1 / 3}$.

Under the assumption of Theorem 3.1. we find two $\rho_{0}$ 's that solve the equation $\rho_{0}^{2}+\tau /\left(\rho_{0} f^{\prime}(0)\right)=$ $m_{0}$. The smaller $\rho_{0}$ is less than $\left(\tau /\left(2 f^{\prime}(0)\right)\right)^{1 / 3}$ and the larger $\rho_{0}$ is greater than $\left(\tau /\left(2 f^{\prime}(0)\right)\right)^{1 / 3}$. From these two $\rho_{0}$ 's we deduce that there exist two $\rho$ 's of the form $\epsilon^{2 / 3} \rho_{0}+o\left(\epsilon^{2 / 3}\right)$ such that 3.1 , holds. These two $\rho$ 's now give rise to two approximate solutions $w$. The $w$ 's nearly solve (1.3) in the following sense.

LEMmA 3.4 There exists $\delta>0$ independent of $\epsilon$ such that $\|S(w)\|_{\infty}=O\left(e^{-(1+\delta) \sqrt{f^{\prime}(z)} / \epsilon}\right)$.

Proof. Let $\tilde{v}=v-z$. Define $h(\tilde{v})$ by

$$
f(v)=f(z+\tilde{v})=f(z)+f^{\prime}(z) \tilde{v}+h(\tilde{v}) .
$$

In the special case $f(u)=u(u-1 / 2)(u-1)$,

$$
h(\tilde{v})=\frac{f^{\prime \prime}(z)}{2} \tilde{v}^{2}+\frac{f^{\prime \prime \prime}(z)}{6} \tilde{v}^{3}
$$

Then we have

$$
\begin{aligned}
-\epsilon^{2} \Delta w+f(w) & =\beta-f(v)-f^{\prime}(z) g+f(v+g)=\beta-f(z+\tilde{v})-f^{\prime}(z) g+f(z+\tilde{v}+g) \\
& =\beta+h(\tilde{v}+g)-h(\tilde{v})=\beta+h^{\prime}(\tilde{v}) g+O\left(\|g\|_{\infty}^{2}\right) \\
& =\beta+O\left(\|\tilde{v} g\|_{\infty}\right)+O\left(\|g\|_{\infty}^{2}\right)=\beta+O\left(e^{-(1+\delta) \sqrt{f^{\prime}(z)} / \epsilon}\right)
\end{aligned}
$$

for some $\delta>0$ by Lemmas 2.1 and 3.2. To reach the last line, we note that

$$
\tilde{v}(r)=O\left(e^{-\sqrt{f^{\prime}(z)} r / \epsilon}\right), \quad g(r)=O\left(e^{-\sqrt{f^{\prime}(z)} / \epsilon}\right)
$$

if $r>1-\iota$ for a small $\iota$; and if $r \leqslant 1-\iota$, then

$$
g(r)=-v^{\prime}(1) \hat{g}(r)=O\left(e^{-\sqrt{f^{\prime}(z)} / \epsilon}\right) O\left(e^{-\delta_{1} / \epsilon}\right)
$$

for some $\delta_{1}>0$, since $\hat{g}(r)=O\left(e^{-\delta_{1} / \epsilon}\right)$ there. We are now left with $\overline{f(w)}$. Since $S(w)=$ $-\epsilon^{2} \Delta w+f(w)-\overline{f(w)}$ and $\overline{S(w)}=0$, we find

$$
\overline{f(w)}=\overline{-\epsilon^{2} \Delta w+f(w)}=\beta+O\left(e^{-(1+\delta) \sqrt{f^{\prime}(z)} / \epsilon}\right) .
$$

Therefore

$$
S(w)=O\left(e^{-(1+\delta) \sqrt{f^{\prime}(z)} / \epsilon}\right) .
$$

In the second step we look for exact solutions. Take one of the two approximate solutions. Denote it by $w$ with $w=v+g$. Next $\rho$ (hence $z$ and $\beta$ ) is chosen so that $\bar{w}=m$, and $\rho_{0}$ satisfies the equation in Lemma 3.3 . We define two function spaces

$$
\begin{aligned}
& \mathcal{X}=\left\{u \in W^{2,2}(\Omega): u=u(|x|), \partial_{\nu} u=0 \text { on } \partial \Omega, \bar{u}=m\right\}, \\
& \mathcal{Y}=\left\{q \in L^{2}(\Omega): q=q(|x|), \bar{q}=0\right\} .
\end{aligned}
$$

The nonlinear operator $S$ maps $\mathcal{X}$ to $\mathcal{Y}$. 
We look for a solution of $S(u)=0$ of the form $w+\phi_{*}$ where $\phi_{*}$ is a small correction to the approximate solution $w$. It is in the function space

$$
\mathcal{F}=\left\{\phi \in W^{2,2}(\Omega): \phi=\phi(|x|), \partial_{\nu} \phi=0 \text { on } \partial \Omega, \bar{\phi}=0\right\} .
$$

Rewrite $S\left(w+\phi_{*}\right)=0$ as

$$
S(w)+L\left(\phi_{*}\right)+R\left(\phi_{*}\right)=0 .
$$

In (3.2), $L$ is the linearized operator of $S$ at $w$ :

$$
L(\phi):=-\epsilon^{2} \Delta \phi+f^{\prime}(w) \phi-\overline{f^{\prime}(w) \phi}, \quad \phi \in \mathcal{F} .
$$

The last term in 3.2 defines the remainder

$$
R(\phi):=f(w+\phi)-f(w)-f^{\prime}(w) \phi-\overline{f(w+\phi)-f(w)-f^{\prime}(w) \phi} .
$$

It turns out that the operator $L$ is invertible. The spectrum of $L$ is bounded away from 0 by a distance of order $\epsilon^{4 / 3}$.

Lemma 3.5 The operator $L: \mathcal{F} \rightarrow \mathcal{Y}$ is one-to-one and onto. There exists a constant $C$ independent of small $\epsilon$ so that $\|\phi\|_{\infty} \leqslant C \epsilon^{-4 / 3}\|L(\phi)\|_{\infty}$ for all $\phi \in \mathcal{F}$.

The proof of this lemma is quite long, so we leave it to Appendix A. Rewrite 3.2 in a fixed point form

$$
\phi_{*}=L^{-1}\left(-S(w)-R\left(\phi_{*}\right)\right) .
$$

Hence we define a nonlinear operator $T$ by

$$
T(\phi)=L^{-1}(-S(w)-R(\phi)) .
$$

We set the domain of $T$ to be

$$
\mathcal{D}=\left\{\phi \in L^{\infty}(\Omega): \phi=\phi(|x|), \bar{\phi}=0,\|\phi\|_{\infty} \leqslant e^{-\left(1+\delta_{2}\right) \sqrt{f^{\prime}(z)} / \epsilon}\right\}
$$

where $\delta_{2}$ is any positive number independent of $\epsilon$ and less than the $\delta$ of Lemma 3.4 Note that we use the $L^{\infty}$ norm in $\mathcal{D}$.

Lemma 3.6 The operator $T$ on $\mathcal{D}$ is a contraction map. There is a unique fixed point $\phi_{*}$.

Proof. From Lemmas 3.4 and 3.5 we deduce

$$
\|T(\phi)\|_{\infty} \leqslant C \epsilon^{-4 / 3}\left(\|S(w)\|_{\infty}+\|R(\phi)\|_{\infty}\right) \leqslant C \epsilon^{-4 / 3}\left(O\left(e^{-(1+\delta) \sqrt{f^{\prime}(z)} / \epsilon}\right)+\|\phi\|_{\infty}^{2}\right) .
$$

Hence $T$ maps $\mathcal{D}$ to itself if $\epsilon$ is sufficiently small. For two $\phi_{1}$ and $\phi_{2}$ in $\mathcal{D}$,

$$
\begin{aligned}
\left\|T\left(\phi_{1}\right)-T\left(\phi_{2}\right)\right\|_{\infty} & \leqslant C \epsilon^{-4 / 3}\left\|R\left(\phi_{1}\right)-R\left(\phi_{2}\right)\right\|_{\infty} \\
& \leqslant C \epsilon^{-4 / 3}\left(\left\|\phi_{1}\right\|_{\infty}+\left\|\phi_{2}\right\|_{\infty}\right)\left\|\phi_{1}-\phi_{2}\right\|_{\infty} \\
& \leqslant C \epsilon^{-4 / 3} e^{-\left(1+\delta_{2}\right) \sqrt{f^{\prime}(z)} / \epsilon}\left\|\phi_{1}-\phi_{2}\right\|_{\infty} .
\end{aligned}
$$

Therefore $T$ is a contraction map when $\epsilon$ is small. A fixed point $\phi_{*}$ exists in $\mathcal{D}$.

The proof of Theorem 3.1 is complete. 


\section{The critical eigenvalues}

Let $u$ be one of the two droplet solutions given in Theorem 3.1. The linearized operator is

$$
L \phi:=-\epsilon^{2} \Delta \phi+f^{\prime}(u) \phi-\overline{f^{\prime}(u) \phi} .
$$

This $L$ differs slightly from the one considered in the last section, for the one there is linearized around $w$. However, the difference between $u$ and $w$ is the exponentially small function $\phi_{*}$, which is a rather insignificant quantity in this section. The stability of $u$ is determined by solving the eigenvalue problem

$$
L \phi=\lambda \phi, \quad \partial_{\nu} \phi=0 \quad \text { on } \partial \Omega, \quad \bar{\phi}=0 .
$$

We first study this eigenvalue problem in the class of radial functions.

THEOREM 4.1 The linear operator $L$, acting on radial functions, has one eigenvalue equal to

$$
\epsilon^{4 / 3}\left(\frac{2 f^{\prime}(0) \rho_{0}}{\tau}-\frac{1}{\rho_{0}^{2}}\right)+o\left(\epsilon^{4 / 3}\right),
$$

which determines the stability of the droplet solutions in the radial class. The corresponding eigenfunction is, up to a constant multiple,

$$
H^{\prime}+\epsilon^{2 / 3} P^{\prime}-\overline{H^{\prime}+\epsilon^{2 / 3} P^{\prime}}+O\left(\epsilon^{4 / 3}\right)
$$

where $H$ and $P$ are given in $2.3,2.4$. Other eigenvalues in the radial class are greater than a positive number that is independent of $\epsilon$. The smaller droplet solution is unstable and the larger droplet solution is stable in the radial class.

The proof mimics the work in [16]. Several ideas have already appeared in the proof of Lemma 3.5. We give an outline of the proof in Appendix B.

Theorem 4.1 only addresses the stability of the droplet solutions in the radial class. To study the stability in the nonradial class, we may separate variables in the equation $L \phi=\lambda \phi$, this time for nonradial $\phi$. For each $j=1,2, \ldots$ there are two independent eigenfunctions $\phi=\zeta(r) \cos (j \theta)$ and $\phi=\zeta(r) \sin (j \theta)$. The radially symmetric function $\zeta$ is a solution of the equation

$$
-\epsilon^{2} \zeta_{r r}-\frac{\epsilon^{2}}{r} \zeta_{r}+\frac{\epsilon^{2} j^{2}}{r^{2}} \zeta+f^{\prime}(u) \zeta=\lambda \zeta, \quad \zeta_{r}(1)=0 .
$$

Arguing as in the proof of Theorem 4.1 we may show the following asymptotic expansions for the eigenvalues and eigenfunctions.

THEOREM 4.2 For each $j=1,2, \ldots$ there is an eigenvalue equal to

$$
\frac{\epsilon^{4 / 3}\left(j^{2}-1\right)}{\rho_{0}^{2}}+o\left(\epsilon^{4 / 3}\right) .
$$

To this eigenvalue there correspond two independent eigenfunctions $\zeta(r) \cos (j \theta)$ and $\zeta(r) \sin (j \theta)$ where, up to a constant multiple, $\zeta$ is equal to

$$
H^{\prime}+\epsilon^{2 / 3} P^{\prime}+O\left(\epsilon^{4 / 3}\right)
$$

where $H$ and $P$ are given in 2.3, 2.4. Other eigenvalues are greater than a positive number that is independent of $\epsilon$. 
One sees from this theorem that the eigenvalues corresponding to $j \geqslant 2$ are all of order $\epsilon^{4 / 3}$ and positive. So with respect to these modes both droplet solutions are stable. However, when $j=1$, we have an eigenvalue of higher order $o\left(\epsilon^{4 / 3}\right)$. The theorem does not tell us whether this eigenvalue is positive or negative, i.e. we do not know whether the droplet solutions are stable with respect to the $j=1$ mode. We will return to this issue later.

\section{The general domain}

The main result we will prove here is the analogy of Theorem 3.1 in a general bounded and smooth domain $\Omega$.

THEOREM 5.1 In a general domain $\Omega$, if the mass constraint $m=\epsilon^{2 / 3} m_{0}+o\left(\epsilon^{2 / 3}\right)$ is above the critical level, i.e.

$$
m_{0}>3\left(\frac{\tau}{2 f^{\prime}(0)}\right)^{2 / 3}\left(\frac{\pi}{|\Omega|}\right)^{1 / 3}
$$

then there exist two droplet solutions.

The construction of droplet solutions in a general domain is more complex. We do expect that the spectral properties obtained in Theorems 4.1 and 4.2 remain more or less valid even if $\Omega$ is not a disc. But we cannot restrict ourselves to radial functions. Without the radial symmetry in addition to the small eigenvalue corresponding to the one in Theorem 4.1, the small eigenvalues, corresponding to the ones in Theorem 4.2. have to be considered as well. The small eigenvalues fall into two scales. The one in Theorem 4.1 and the ones in Theorem 4.2 with $j \geqslant 2$ are of order $\epsilon^{4 / 3}$. Their absolute values are considerably greater than that of the one in Theorem 4.2 with $j=1$, which is of order $o\left(\epsilon^{4 / 3}\right)$. The exact size of the latter eigenvalue will be discussed near the end of this section. Our construction of two droplet solutions in a general domain must take this scale difference into consideration.

Let us give an outline of our approach. The reader must be aware that although the notations used in the rest of this paper look similar to the ones used in the earlier sections, we are taking a significantly different approach. We define two function spaces

$$
\mathcal{X}=\left\{u \in W^{2,2}(\Omega): \partial_{\nu} u=0 \text { on } \partial \Omega, \bar{u}=m\right\}, \quad \mathcal{Y}=\left\{q \in L^{2}(\Omega): \bar{q}=0\right\}
$$

and the nonlinear operator $S$ given in 1.4 maps $\mathcal{X}$ to $\mathcal{Y}$. Note that $\mathcal{X}$ and $\mathcal{Y}$ differ from the corresponding spaces in Section 3 in that here the functions in these spaces are generally not radially symmetric.

We first construct a good approximate solution of a droplet, centered at a point $\xi$. $\xi$ must have some distance from $\partial \Omega$. Let $\sigma>0$ be independent of $\epsilon$ and

$$
\Omega_{\sigma}=\{\xi \in \Omega: d \xi>5 \sigma\}
$$

where $d_{\xi}$ is the distance of $\xi$ to $\partial \Omega$. At each $\xi$ we construct an approximate solution whose droplet is centered at $\xi$. This $\xi$ is first an arbitrary point in $\Omega_{\sigma}$. It will be determined in the last step. The constant $\sigma$ is chosen to be sufficiently small so that

$$
\max \left\{d_{\xi}: \xi \in \Omega \backslash \Omega_{\sigma}\right\}<\max \left\{d_{\xi}: \xi \in \Omega\right\}
$$


This ensures that a point in $\Omega$ with the largest distance to $\partial \Omega$ is in $\Omega_{\sigma}$. The choice of the number 5 in 5.1 will be explained in the proof of Lemma 5.5. All estimates in this section are uniform in $\xi \in \Omega_{\sigma}$.

Denote the approximate solution by $w_{\xi}$. As $\xi$ varies in $\Omega_{\sigma}$, the $w_{\xi}$ form a two-dimensional manifold in $\mathcal{X}$ which we denote by

$$
\mathcal{M}=\left\{w_{\xi}: \xi \in \Omega_{\sigma}\right\}
$$

At each point $w_{\xi}$ we define an approximate tangent plane to $\mathcal{M}$ spanned by two functions $b_{1, \xi}$ and $b_{2, \xi}$ that are essentially the truncated versions of the two eigenfunctions of mode $j=1$ studied in Theorem 4.2 Perpendicular to $b_{1, \xi}$ and $b_{2, \xi}$ is the space $\mathcal{F}_{\xi}$ that is almost normal to the surface $\mathcal{M}$.

Next we "solve" $S(u)=0$ in each $\mathcal{F}_{\xi}$ direction. More precisely, we look for a correction function $\phi_{\xi}$ so that

$$
S\left(w_{\xi}+\phi_{\xi}\right)=c_{1} b_{1, \xi}+c_{2} b_{2, \xi}
$$

for some $c_{1}, c_{2} \in \mathbb{R}$. Now we have a second manifold

$$
\mathcal{N}=\left\{w_{\xi}+\phi_{\xi}: \xi \in \Omega_{\sigma}\right\}
$$

of improved approximate solutions.

In the last step we find an exact solution in $\mathcal{N}$. To do this we maximize $I\left(w_{\xi}+\phi_{\xi}\right)$ in $\mathcal{N}$ :

$$
\max \left\{I\left(w_{\xi}+\phi_{\xi}\right): \xi \in \Omega_{\sigma}\right\} .
$$

We will show that the maximizer exists at an interior point of $\Omega$. Actually we will show this maximizer has almost the greatest distance from $\partial \Omega$, among all the points in $\Omega$.

These three steps 5.3, 5.4, 5.6 are carried out in the rest of this section. It turns out that in constructing 5.3 we can find two approximate solutions $w_{\xi}$ at any fixed point $\xi$. One corresponds to a smaller droplet and the other to a larger droplet. Starting with the two approximate solutions and completing the three steps, we will find two droplet solutions in the general domain.

We first recall the profile of a droplet: $v(r)$ given in 2.1). It is a radially symmetric function that decays to $z$ as $r \rightarrow \infty$. Note that $f(z)=\beta$. Define

$$
\tilde{v}=v-z .
$$

Note that $\tilde{v}$ decays to 0 at infinity. It satisfies the equation

$$
-\epsilon^{2} \Delta \tilde{v}+f^{\prime}(z) \tilde{v}+h(\tilde{v})=0
$$

where

$$
h(\tilde{v})=f(v)-f(z)-f^{\prime}(z)(v-z) .
$$

In the particular case $f(u)=u(u-1 / 2)(u-1)$,

$$
h(\tilde{v})=\frac{f^{\prime \prime}(z)}{2} \tilde{v}^{2}+\frac{f^{\prime \prime \prime}(z)}{6} \tilde{v}^{3} .
$$

We need to choose $z$ properly to reflect the mass constraint of the Cahn-Hilliard problem. We look for $z$ so that $z$ and the corresponding $v$ (and $\tilde{v}$ ) determined from $z$ satisfy the relation

$$
f^{\prime}(z)(m-z)|\Omega|+\int_{\mathbb{R}^{2}} h(\tilde{v}) \mathrm{d} x=0 .
$$


Let us explain how we arrive at 5.8). Shift $v$ to $v(\cdot-\xi)$. Integrate 5.7) over $\Omega$ to derive

$$
-\epsilon^{2} \int_{\partial \Omega} \frac{\partial \tilde{v}(\cdot-\xi)}{\partial v} \mathrm{~d} s+f^{\prime}(z) \int_{\Omega} \tilde{v}(\cdot-\xi) \mathrm{d} x+\int_{\Omega} h(\tilde{v}(\cdot-\xi)) \mathrm{d} x=0 .
$$

We ignore the first term on the left side since it is very small. We replace the last term by $\int_{\mathbb{R}^{2}} h(\tilde{v}) \mathrm{d} x$. Regarding the mass constraint we must have $\overline{v(\cdot-\xi)} \approx m$ in $\Omega$, i.e.

$$
\int_{\Omega} \tilde{v}(\cdot-\xi) \mathrm{d} x \approx(m-z)|\Omega| .
$$

After these replacements, we obtain (5.8). Note that $z$ defined in this way is independent of the choice of the center $\xi$ of the droplet.

Lemma 5.2 When $m$ is above the critical level, (5.8) has two solutions of $z$. More precisely, let $z=\epsilon^{2 / 3} z_{0}+o\left(\epsilon^{2 / 3}\right)$ with $z_{0}$ independent of $\epsilon$. Then $z_{0}$ satisfies the condition

$$
z_{0}|\Omega|+\frac{\pi \tau^{2}}{f^{\prime}(0)^{2} z_{0}^{2}}=m_{0}|\Omega|, \quad \text { where } \quad m=\epsilon^{2 / 3} m_{0}+o\left(\epsilon^{2 / 3}\right) .
$$

Proof. The equation (5.8) implies that

$$
f^{\prime}(z)(m-z)|\Omega|+h(1) \pi \rho^{2}+o\left(\epsilon^{2 / 3}\right)=0 .
$$

The lemma follows once we note that $h(1)=-f^{\prime}(0)+o(1)$ and $\rho_{0}=\tau /\left(f^{\prime}(0) z_{0}\right)$ in view of $f^{\prime}(0) z_{0}=\beta_{0}$ and Lemma 2.3 .

Here one solution $z$ corresponds to a smaller droplet and the other to a larger droplet. In terms of $\rho$ (recall that $v(\rho)=1 / 2$ ) this lemma says the following.

Lemma 5.3 Suppose $m=\epsilon^{2 / 3} m_{0}+o\left(\epsilon^{2 / 3}\right)$ and $\rho=\epsilon^{1 / 3} \rho_{0}+o\left(\epsilon^{1 / 3}\right)$. Then $\rho_{0}$ and $m_{0}$ must satisfy the equation

$$
\frac{\pi}{|\Omega|} \rho_{0}^{2}+\frac{\tau}{\rho_{0} f^{\prime}(0)}=m_{0} .
$$

Therefore $m_{0}$ cannot be less than $3\left(\tau /\left(2 f^{\prime}(0)\right)\right)^{2 / 3}(\pi /|\Omega|)^{1 / 3}$. This last value for $m_{0}$ is attained if $\rho_{0}=\left(|\Omega| \tau /\left(2 \pi f^{\prime}(0)\right)\right)^{1 / 3}$.

Proof. Use the relations $\beta_{0}=f^{\prime}(0) z_{0}$ and $\beta_{0}=\tau / \rho_{0}$ from Lemma 2.3 to rewrite the equation in Lemma 5.2 in terms of $\rho_{0}$ instead of $z_{0}$.

Now we move $v$ to $v(\cdot-\xi)$ so that the center of the droplet is at an arbitrary point $\xi \in \Omega_{\sigma}$. This $v(\cdot-\xi)$ does not satisfy the Neumann boundary condition. We introduce $g_{\xi}$ which is the solution of the linear problem

$$
-\epsilon^{2} \Delta g_{\xi}+f^{\prime}(z) g_{\xi}=0 \quad \text { in } \Omega, \quad \partial_{\nu} g_{\xi}=-\partial_{\nu} v(\cdot-\xi) \quad \text { on } \partial \Omega .
$$

Then $v(\cdot-\xi)+g_{\xi}$ satisfies the Neumann boundary condition. Finally, to have the mass constraint satisfied we introduce a number $\eta_{\xi}$ so that

$$
w_{\xi}=v(\cdot-\xi)+g_{\xi}+\eta_{\xi} \quad \text { and } \quad \bar{w}_{\xi}=m .
$$

Here $w_{\xi}$ is our approximate solution, from which we obtain the manifold $\mathcal{M}(5.3)$, in $\mathcal{X}$.

The properties of $w_{\xi}$ are given in the following lemma. We leave its rather technical proof to Appendix C. 
LEMMA 5.4 Let the distance from $\xi \in \Omega_{\sigma}$ to $\partial \Omega$ be $d_{\xi}=\min \{|x-\xi|: x \in \partial \Omega\}$.

(1) $\left\|S\left(w_{\xi}\right)\right\|_{L^{2}(\Omega)}=O\left(e^{-(1+\delta) \sqrt{f^{\prime}(z)} d_{\xi} / \epsilon}\right)$ for some small $\delta>0$ independent of $\epsilon$.

(2) There exist constants $C_{0}, C_{1}, a_{0}$ and $a_{1}$ independent of $\epsilon$ and $\xi$, and a constant $C_{\epsilon}$ independent of $\xi$ but depending on $\epsilon$ so that

$$
C_{\epsilon}-C_{0} e^{a_{0} \epsilon^{-2 / 3}} e^{-2 \sqrt{f^{\prime}(z)} d_{\xi} / \epsilon} \leqslant I\left(w_{\xi}\right) \leqslant C_{\epsilon}-C_{1} e^{-a_{1} \epsilon^{-2 / 3}} e^{-2 \sqrt{f^{\prime}(z)} d_{\xi} / \epsilon} .
$$

When we keep track of the decay rate of $I\left(w_{\xi}\right)$ to $C_{\epsilon}$, the dominating part is $e^{-2 \sqrt{f^{\prime}(z)} d_{\xi} / \epsilon}$. Both $e^{a_{0} \epsilon^{-2 / 3}}$ and $e^{-a_{1} \epsilon^{-2 / 3}}$ are rather negligible.

Now that we have a family of approximate solutions, we proceed to solve (5.4). It is sometimes more convenient to work with the rescaled domain. Let $\Omega_{\xi}=\left\{y \in \mathbb{R}^{2}: \epsilon y+\xi \in \Omega\right\}$. Note that $\Omega_{\xi}$ is a large domain that depends on $\epsilon$ as well as $\xi$. The $L^{2}$ and $W^{2,2}$ norms on the rescaled domain $\Omega \xi$ are more appropriate for our problem than the corresponding norms on $\Omega$. For simplicity we will write $\phi(y)=\phi(x)$ with $x=\epsilon y+\xi$. In the following, differentiation, as in the Laplace operator, is taken with respect to $y$.

At each $w_{\xi}$ we define an approximate tangent plane to $\mathcal{M}$. Recall the two eigenfunctions associated with eigenvalue $\lambda_{1}$ studied in Theorem 4.2. They are of the form

$$
\left(H^{\prime}+\epsilon^{2 / 3} P^{\prime}+O\left(\epsilon^{4 / 3}\right)\right) \cos \theta \text { and }\left(H^{\prime}+\epsilon^{2 / 3} P^{\prime}+O\left(\epsilon^{4 / 3}\right)\right) \sin \theta .
$$

We shift the center of these functions to $\xi$ so that $r=|x-\xi|$ and $\theta=\arctan \left(\left(x_{2}-\xi_{2}\right) /\left(x_{1}-\xi_{1}\right)\right)$. The radial parts of these two functions decay exponentially fast. We truncate the exponentially small tails of $H^{\prime}+\epsilon^{2 / 3} Q^{\prime}+O\left(\epsilon^{4 / 3}\right)$ to define

$$
b_{1, \xi}=\left(H^{\prime}+\epsilon^{2 / 3} P^{\prime}+O\left(\epsilon^{4 / 3}\right)\right) \cos \theta, \quad b_{2, \xi}=\left(H^{\prime}+\epsilon^{2 / 3} P^{\prime}+O\left(\epsilon^{4 / 3}\right)\right) \sin \theta,
$$

which have compact support in $\Omega$. More precisely, the supports of $b_{1, \xi}$ and $b_{2, \xi}$ must be in $B_{\sigma}(\xi)$ where $\sigma$ is given after 5.1 .

At each $w_{\xi}$ of the manifold $\mathcal{M}$ we define

$$
\mathcal{F}_{\xi}=\left\{\phi \in W^{2,2}\left(\Omega_{\xi}\right): \bar{\phi}=0, \partial_{\nu} \phi=0 \text { on } \partial \Omega_{\xi}, \phi \perp b_{j, \xi}, j=1,2\right\}
$$

where $\perp$ is defined from the $L^{2}\left(\Omega_{\xi}\right)$ inner product. Note that in the rescaled domain $\Omega_{\xi}, \bar{\phi}$ is the average of $\phi$ over $\Omega_{\xi}$. Then $w_{\xi}+\mathcal{F}_{\xi}$ is a subset of $\left\{u \in W^{2,2}\left(\Omega_{\xi}\right): \partial_{\nu} u=0\right.$ on $\left.\partial \Omega_{\xi}, \bar{u}=m\right\}$, which we call the $\xi$-fiber of $\mathcal{M}$. Define

$$
\mathcal{E}_{\xi}=\left\{q \in L^{2}\left(\Omega_{\xi}\right): \bar{q}=0, q \perp b_{j, \xi}, j=1,2\right\},
$$

which is a subspace of $\left\{q \in L^{2}\left(\Omega_{\xi}\right): \bar{q}=0\right\}$. Let the projection to $\mathcal{E}_{\xi}$ be

$$
\pi_{\xi}:\left\{q \in L^{2}\left(\Omega_{\xi}\right): \bar{q}=0\right\} \rightarrow \mathcal{E}_{\xi} .
$$

To solve (5.4) we look for a $\phi_{\xi} \in \mathcal{F}_{\xi}$ so that

$$
\pi_{\xi} \circ S\left(w_{\xi}+\phi_{\xi}\right)=0 .
$$

For each $\phi \in \mathcal{F}_{\xi}$ we expand

$$
S\left(w_{\xi}+\phi\right)=S\left(w_{\xi}\right)+L_{\xi}(\phi)+R_{\xi}(\phi)
$$


where

$$
L_{\xi}(\phi)=-\Delta \phi+f^{\prime}\left(w_{\xi}\right) \phi-\overline{f^{\prime}\left(w_{\xi}\right) \phi}
$$

is the linearization of $S$ at $w_{\xi}$, and

$$
R_{\xi}(\phi)=f\left(w_{\xi}+\phi\right)-f\left(w_{\xi}\right)-f^{\prime}\left(w_{\xi}\right) \phi-\overline{f\left(w_{\xi}+\phi\right)-f\left(w_{\xi}\right)-f^{\prime}\left(w_{\xi}\right) \phi}
$$

Note that when we use the rescaled variable $y$, there is no $\epsilon^{2}$ in front of $\Delta$ in $L_{\xi}$. Then 5.10 , is written as

$$
\pi_{\xi} \circ S\left(w_{\xi}\right)+\pi_{\xi} \circ L_{\xi}\left(\phi_{\xi}\right)+\pi_{\xi} \circ R_{\xi}\left(\phi_{\xi}\right)=0 .
$$

Regarding the linear operator $\pi_{\xi} \circ L_{\xi}: \mathcal{F}_{\xi} \rightarrow \mathcal{E}_{\xi}$ we have the following lemma.

LEMmA 5.5 The operator $\pi_{\xi} \circ L_{\xi}$ is one-to-one from $\mathcal{F}_{\xi}$ onto $\mathcal{E}_{\xi}$. There exists $C>0$ independent of $\epsilon$ such that $\|\phi\|_{W^{2,2}\left(\Omega_{\xi}\right)} \leqslant C \epsilon^{-4 / 3}\left\|\pi_{\xi} \circ L_{\xi}(\phi)\right\|_{L^{2}\left(\Omega_{\xi}\right)}$ for all $\phi \in \mathcal{F}_{\xi}$.

The proof of this lemma is difficult. We leave it to Appendix D. Lemma 5.5 gives a measurement of the invertibility of $\pi_{\xi} \circ L_{\xi}$. The equation 5.11) can now be solved by a fixed point argument.

LEMMA 5.6 There exists $\phi_{\xi} \in \mathcal{F}_{\xi}$ so that $\pi_{\xi} \circ S_{\xi}\left(w_{\xi}+\phi_{\xi}\right)=0$. Moreover,

$$
\left\|\phi_{\xi}\right\|_{W^{2,2}\left(\Omega_{\xi}\right)}=O\left(e^{-(1+\delta) \sqrt{f^{\prime}(z)} d_{\xi} / \epsilon}\right)
$$

for some small $\delta>0$ independent of $\epsilon$.

Proof. We write (5.11) in a fixed point form:

$$
\phi_{\xi}=\left(\pi_{\xi} \circ L_{\xi}\right)^{-1}\left(-\pi_{\xi} \circ S\left(w_{\xi}\right)-\pi_{\xi} \circ R_{\xi}\left(\phi_{\xi}\right)\right) .
$$

We define the operator $T_{\xi}$ from $\mathcal{D}_{\xi}$ to itself by

$$
T_{\xi}(\phi)=\left(\pi_{\xi} \circ L_{\xi}\right)^{-1}\left(-\pi_{\xi} \circ S\left(w_{\xi}\right)-\pi_{\xi} \circ R_{\xi}(\phi)\right)
$$

where

$$
\mathcal{D}_{\xi}=\left\{\phi \in W^{2,2}\left(\Omega_{\xi}\right): \bar{\phi}=0, \phi \perp b_{j, \xi}, j=1,2\right\} .
$$

By Lemma 5.4 (1), on the rescaled domain $\Omega_{\xi}$ we have

$$
\left\|S\left(w_{\xi}\right)\right\|_{L^{2}\left(\Omega_{\xi}\right)}=O\left(\epsilon^{-1} e^{-(1+\delta) \sqrt{f^{\prime}(z)} d_{\xi} / \epsilon}\right) .
$$

Let $\mathcal{B}_{\xi}$ be a closed ball in $\mathcal{D}_{\xi}$ defined by

$$
\mathcal{B}_{\xi}=\left\{\phi \in \mathcal{D}_{\xi}:\|\phi\|_{W^{2,2}\left(\Omega_{\xi}\right)} \leqslant C_{1} \epsilon^{-7 / 3} e^{-(1+\delta) \sqrt{f^{\prime}(z)} d_{\xi} / \epsilon}\right\}
$$

where $C_{1}$ is a constant independent of $\epsilon$ to be determined soon. Then for every $\phi \in \mathcal{B}_{\xi}$,

$$
\begin{aligned}
\left\|T_{\xi}(\phi)\right\|_{W^{2,2}\left(\Omega_{\xi}\right)} & \leqslant \epsilon^{-4 / 3}\left(\left\|\pi_{\xi} \circ S\left(w_{\xi}\right)\right\|_{L^{2}\left(\Omega_{\xi}\right)}+\left\|\pi_{\xi} \circ R(\phi)\right\|_{L^{2}\left(\Omega_{\xi}\right)}\right) \\
& \leqslant \epsilon^{-4 / 3} O\left(\epsilon^{-1} e^{-(1+\delta) \sqrt{f^{\prime}(z)} d_{\xi} / \epsilon}\right)+C \epsilon^{-4 / 3}\|\phi\|_{L^{4}\left(\Omega_{\xi}\right)}^{2} \\
& \leqslant O\left(\epsilon^{-7 / 3} e^{-(1+\delta) \sqrt{f^{\prime}(z)} d_{\xi} / \epsilon}\right)+C \epsilon^{-4 / 3}\|\phi\|_{W^{2,2}\left(\Omega_{\xi}\right)}^{2} \\
& \leqslant O\left(\epsilon^{-7 / 3} e^{-(1+\delta) \sqrt{f^{\prime}(z)} d_{\xi} / \epsilon}\right)+C \epsilon^{-4 / 3}\left[C_{1} \epsilon^{-7 / 3} e^{-(1+\delta) \sqrt{f^{\prime}(z)} d_{\xi} / \epsilon}\right]^{2}
\end{aligned}
$$


where $C$ is a constant and we have used the Sobolev embedding theorem. We see that if we choose $C_{1}$ to be sufficiently large, then $T_{\xi}$ maps $\mathcal{B}_{\xi}$ into itself. As in the proof of Lemma 3.6 we can similarly show that this mapping is a contraction. Then by the contraction mapping theorem we conclude that there is a fixed point $\phi_{\xi}$. Since $\phi_{\xi} \in \mathcal{D}_{\xi}$, we have

$$
\left\|\phi_{\xi}\right\|_{W^{2,2}\left(\Omega_{\xi}\right)}=O\left(\epsilon^{-7 / 3} e^{-(1+\delta) \sqrt{f^{\prime}(z)} d_{\xi} / \epsilon}\right) .
$$

By changing $\delta$ to a smaller value we obtain

$$
\left\|\phi_{\xi}\right\|_{W^{2,2}\left(\Omega_{\xi}\right)}=O\left(e^{-(1+\delta) \sqrt{f^{\prime}(z)} d_{\xi} / \epsilon}\right) .
$$

In the final step we look for a particular $\xi_{*}$ so that $\xi_{*}$ maximizes $I\left(w_{\xi}+\phi_{\xi}\right)$ with respect to $\xi$ and consequently $S\left(w_{\xi_{*}}+\phi_{\xi_{*}}\right)=0$. To this end we first show

LEMMA 5.7

$$
I\left(w_{\xi}+\phi_{\xi}\right)=I\left(w_{\xi}\right)+O\left(e^{-(2+\delta) \sqrt{f^{\prime}(z)} d_{\xi} / \epsilon}\right)
$$

for some $\delta>0$.

Proof. Let

$$
R_{\xi, 1}\left(\phi_{\xi}\right)=F\left(w_{\xi}+\phi_{\xi}\right)-F\left(w_{\xi}\right)-f\left(w_{\xi}\right) \phi_{\xi}-\frac{1}{2} f^{\prime}\left(w_{\xi}\right) \phi_{\xi}^{2}
$$

We expand $I\left(w_{\xi}+\phi_{\xi}\right)$ as follows:

$$
I\left(w_{\xi}+\phi_{\xi}\right)=I\left(w_{\xi}\right)+\int_{\Omega} S\left(w_{\xi}\right) \phi_{\xi} \mathrm{d} x+\frac{1}{2} \int_{\Omega} L_{\xi}\left(\phi_{\xi}\right) \phi_{\xi} \mathrm{d} x+\int_{\Omega} R_{\xi, 1}\left(\phi_{\xi}\right) \mathrm{d} x .
$$

Since $S\left(w_{\xi}+\phi_{\xi}\right)=0$, we have

$$
S\left(w_{\xi}\right)+L_{\xi}\left(\phi_{\xi}\right)+R_{\xi, 2}\left(\phi_{\xi}\right)=\text { const }
$$

where

$$
R_{\xi, 2}\left(\phi_{\xi}\right)=f\left(w_{\xi}+\phi_{\xi}\right)-f\left(w_{\xi}\right)-f^{\prime}\left(w_{\xi}\right) \phi_{\xi} .
$$

After substitution we obtain

$$
I\left(w_{\xi}+\phi_{\xi}\right)=I\left(w_{\xi}\right)+\frac{1}{2} \int_{\Omega} S\left(w_{\xi}\right) \phi_{\xi} \mathrm{d} x+\int_{\Omega}\left[R_{\xi, 1}\left(\phi_{\xi}\right)-\frac{1}{2} R_{\xi, 2}\left(\phi_{\xi}\right) \phi_{\xi}\right] \mathrm{d} x .
$$

The third term on the right side is bounded by

$$
\left|\int_{\Omega}\left[R_{\xi, 1}\left(\phi_{\xi}\right)-\frac{1}{2} R_{\xi, 2}\left(\phi_{\xi}\right) \phi_{\xi}\right] \mathrm{d} x\right| \leqslant C \int_{\Omega}\left|\phi_{\xi}\right|^{3} \mathrm{~d} x \leqslant C \epsilon^{2}\left\|\phi_{\xi}\right\|_{W^{2,2}\left(\Omega_{\xi}\right)}^{3}=O\left(e^{-(2+\delta) \sqrt{f^{\prime}(z)} d_{\xi} / \epsilon}\right)
$$

for some $\delta>0$ by Lemma 5.6. For the second term one has

$$
\left|\int_{\Omega} S\left(w_{\xi}\right) \phi_{\xi} \mathrm{d} x\right|=\epsilon^{2}\left|\int_{\Omega_{\xi}} S\left(w_{\xi}\right) \phi_{\xi} \mathrm{d} y\right| \leqslant \epsilon^{2}\left\|S\left(w_{\xi}\right)\right\|_{L^{2}\left(\Omega_{\xi}\right)}\left\|\phi_{\xi}\right\|_{L^{2}\left(\Omega_{\xi}\right)}=O\left(e^{-(2+\delta) \sqrt{f^{\prime}(z)} d_{\xi} / \epsilon}\right)
$$

for some $\delta>0$ by Lemmas 5.4 (1) and 5.6. Lemma 5.7 then follows. 
Combining Lemmas $5.4(2)$ and 5.7 we deduce that $I\left(w_{\xi}+\phi_{\xi}\right)$ and $I\left(w_{\xi}\right)$ have the same asymptotic property:

$$
C_{\epsilon}-C_{0} e^{a_{0} \epsilon^{-2 / 3}} e^{-2 \sqrt{f^{\prime}(z)} d_{\xi} / \epsilon} \leqslant I\left(w_{\xi}+\phi_{\xi}\right) \leqslant C_{\epsilon}-C_{1} e^{a_{1} \epsilon^{-2 / 3}} e^{-2 \sqrt{f^{\prime}(z)} d_{\xi} / \epsilon} .
$$

To maximize $I\left(w_{\xi}+\phi_{\xi}\right)$ we just need to maximize $d_{\xi}$. The maximizer $\xi_{*}$ is exponentially close to a point whose distance to $\partial \Omega$ is the greatest among all $\xi \in \Omega$.

One can then show that $w_{\xi_{*}}+\phi_{\xi_{*}}$ is an exact solution of $S\left(w_{\xi_{*}}+\phi_{\xi_{*}}\right)=0$. The idea is that at $\xi=\xi_{*}$

$$
\left.\frac{\partial I\left(w_{\xi}+\phi_{\xi}\right)}{\partial \xi_{j}}\right|_{\xi=\xi_{*}}=0, \quad j=1,2 .
$$

This implies that $c_{1}=c_{2}=0$ at $\xi=\xi_{*}$ where $c_{1}$ and $c_{2}$ are given in (5.4). This argument is standard and the details can be found, for instance, in [12, Section 5]. The proof of Theorem[5.1 is complete.

A remark about the stability of these two droplet solutions is in order. As in the last section, the smaller droplet solution is unstable. For the larger droplet solution, when we solve the equation $\pi_{\xi} \circ S\left(w_{\xi}+\phi_{\xi}\right)=0$, the solution $w_{\xi}+\phi_{\xi}$ is stable in this step, very much like in the last section where we were restricted to radial functions. However, to find $w_{\xi_{*}}+\phi_{\xi_{*}}$, we maximized $I\left(w_{\xi}+\phi_{\xi}\right)$ with respect to $\xi$. In this step the solution $w_{\xi_{*}}+\phi_{\xi_{*}}$ is unstable. Overall, the larger droplet solution is also unstable. In the last section we were left with the question whether with respect to the $j=1$ mode the larger radial droplet solution is stable. Now we know that the $j=1$ mode is unstable. Moreover, because, as we vary $\xi, I\left(w_{\xi}+\phi_{\xi}\right)$ changes by an exponentially small amount, the eigenvalue of the $j=1$ mode of the last section should be negative but exponentially close to 0 .

\section{Multiple droplets}

We now consider solutions with multiple droplets. Let $K$ be a positive integer. We show the existence of a critical mass constraint, which depends on $K$, so that when the mass is above this critical value, two solutions with multiple droplets exist. Our approach closely follows the argument in the last section. We only emphasize the modifications that are needed while omitting the details that are identical to the ones before.

In the case of single droplet solutions, the center of the droplet is given by $\xi_{*}$ that almost maximizes the distance function $d_{\xi}$ of $\xi \in \Omega$ to $\partial \Omega$. In the multi-droplet case the role of the distance function is played by the function $\varphi\left(\xi^{1}, \ldots, \xi^{K}\right)$ given in 1.5 for any

$$
\left(\xi^{1}, \ldots, \xi^{K}\right) \in \Omega \times \cdots \times \Omega, \quad \xi^{l} \neq \xi^{m} \text { if } l \neq m .
$$

If $\xi^{k} \rightarrow \partial \Omega$ for some $k$ or $\left|\xi^{l}-\xi^{m}\right| \rightarrow 0$ for some $l$ and $m$, then $\varphi\left(\xi^{1}, \ldots, \xi^{K}\right) \rightarrow 0$. Hence $\varphi$ admits a maximum. Maximizing $\varphi\left(\xi^{1}, \ldots, \xi^{K}\right)$ is a disc packing problem. If we place $K$ discs, all of radius $\varphi\left(\xi^{1}, \ldots, \xi^{K}\right)$, centered at $\xi^{k}, k=1, \ldots, K$, these $K$ discs reside inside $\Omega$ and they are mutually disjoint. The maximum value of $\varphi(\xi)$ is the greatest possible radius we can have as we pack the discs.

The main result in this section is the following existence theorem.

THEOREM 6.1 If the mass constraint $m=\epsilon^{2 / 3} m_{0}+o\left(\epsilon^{2 / 3}\right)$ is above the critical level:

$$
m_{0}>3\left(\frac{\tau}{2 f^{\prime}(0)}\right)^{2 / 3}\left(\frac{K \pi}{|\Omega|}\right)^{1 / 3}
$$


then there exist two solutions with $K$ droplets. For each of the two solutions the centers of the droplets $\xi_{*}^{1}, \ldots, \xi_{*}^{K}$ almost maximize the function $\varphi\left(\xi^{1}, \ldots, \xi^{K}\right)$.

For multiple droplet solutions, the critical mass constraint is greater than the critical mass for single droplet solutions. If the critical mass constraint were to be attained, the radius of each droplet would be $\rho=\epsilon^{1 / 3} \rho_{0}+o\left(\epsilon^{1 / 3}\right)$ with $\rho_{0}$ being $\left(|\Omega| \tau /\left(2 K \pi f^{\prime}(0)\right)\right)^{1 / 3}$ which is less than the corresponding value in the single droplet case (see Lemma 6.3 below).

The proof of the theorem is again divided into three steps. First we construct a family of approximate solutions parametrized by $\xi=\left(\xi^{1}, \ldots, \xi^{K}\right)$. We have promoted $\xi$ to a $K$-vector which is an arbitrary member in

$$
\Omega_{\sigma}^{K}=\left\{\left(\xi^{1}, \ldots, \xi^{K}\right) \in \Omega^{K}: d_{\xi^{k}}>5 \sigma,\left|\xi^{k}-\xi^{l}\right|>10 \sigma, k, l=1, \ldots, K\right\} .
$$

Here $\sigma$ is a small positive number independent of $\epsilon$. It is chosen so that any maximum of $\varphi$ is in $\Omega_{\sigma}^{K}$. We will explain in the proof of Lemma 6.5 why we have the numbers 5 and 10 in 6.1 . All estimates in this section are uniform in $\xi \in \Omega_{\sigma}^{K}$.

We use the same function spaces $\mathcal{X}, \mathcal{Y}$ and the nonlinear operator $S$ as in the last section. The droplet profile is again given by $v$ of 2.1 . To determine the value $z$ we solve, instead of (5.8),

$$
f^{\prime}(z)(m-z)|\Omega|+K \int_{\mathbb{R}^{2}} h(\tilde{v})=0 \quad \text { where } \quad \tilde{v}=v-z
$$

LEMMA 6.2 When $m$ is above the critical level, 5.8) has two solutions $z$. Let $z=\epsilon^{2 / 3} z_{0}+o\left(\epsilon^{2 / 3}\right)$ with $z_{0}$ independent of $\epsilon$. Then $z_{0}$ satisfies the condition

$$
z_{0}|\Omega|+\frac{K \pi \tau^{2}}{f^{\prime}(0)^{2} z_{0}^{2}}=m_{0}|\Omega|, \quad \text { where } \quad m=\epsilon^{2 / 3} m_{0}+o\left(\epsilon^{2 / 3}\right) .
$$

Lemma 6.3 Suppose $m=\epsilon^{2 / 3} m_{0}+o\left(\epsilon^{2 / 3}\right)$ and $\rho=\epsilon^{1 / 3} \rho_{0}+o\left(\epsilon^{1 / 3}\right)$. Then $\rho_{0}$ and $m_{0}$ must satisfy the equation

$$
\frac{K \pi}{|\Omega|} \rho_{0}^{2}+\frac{\tau}{\rho_{0} f^{\prime}(0)}=m_{0} .
$$

Therefore $m_{0}$ cannot be less than $3\left(\tau /\left(2 f^{\prime}(0)\right)\right)^{2 / 3}(K \pi /(|\Omega|))^{1 / 3}$. The latter value for $m_{0}$ is attained if $\rho_{0}=\left(|\Omega| \tau /\left(2 K \pi f^{\prime}(0)\right)\right)^{1 / 3}$.

Move $\tilde{v}$ to $\tilde{v}\left(\cdot-\xi^{k}\right)=\tilde{v}_{\xi^{k}}=\tilde{v}_{k}$ for a $\xi^{k} \in \Omega$. Define $g_{k}$ to be the solution of

$$
-\epsilon^{2} \Delta g_{k}+f^{\prime}(z) g_{k}=0, \quad \partial_{\nu} g_{k}=-\partial_{\nu} v\left(\cdot-\xi^{k}\right) \quad \text { on } \partial \Omega
$$

Given $\xi$ we define

$$
\tilde{w}_{\xi}=\sum_{k=1}^{K}\left(\tilde{v}_{k}+g_{k}\right), \quad w_{\xi}=\tilde{w}_{\xi}+z+\eta_{\xi}
$$

where $\eta_{\xi}$ is a number chosen so that $\bar{w}_{\xi}=m$. As we vary $\xi$ in $w_{\xi}$ we obtain a manifold $\mathcal{M}$ of dimension $2 K$ in $\mathcal{X}$. The next lemma generalizes Lemma 5.4 whose proof is left to Appendix E. 
LEMMA 6.4 (1) $\left\|S\left(w_{\xi}\right)\right\|_{L^{2}(\Omega)}=O\left(e^{-(1+\delta) \sqrt{f^{\prime}(z)} \varphi(\xi) / \epsilon}\right)$ for some small $\delta>0$ independent of $\epsilon$. (2) There exist constants $C_{0}, C_{1}, a_{0}$ and $a_{1}$ independent of $\epsilon$ and $\xi$, and a constant $C_{\epsilon}$ independent of $\xi$ but depending on $\epsilon$ so that

$$
C_{\epsilon}-C_{0} e^{a_{0} \epsilon^{-2 / 3}} e^{-2 \sqrt{f^{\prime}(z)} \varphi(\xi) / \epsilon} \leqslant I\left(w_{\xi}\right) \leqslant C_{\epsilon}-C_{1} e^{-a_{1} \epsilon^{-2 / 3}} e^{-2 \sqrt{f^{\prime}(z)} \varphi(\xi) / \epsilon} .
$$

To define the approximate tangent space of $\mathcal{M}$ at $w_{\xi}$, we move the eigenfunctions corresponding to $\lambda_{1}$ in Theorem 4.2 to each $\xi^{k}$. Truncate the radial part so that they have support in $\Omega$. Denote these functions by $b_{1, \xi}^{k}$ and $b_{2, \xi}^{k}(k=1, \ldots, K)$. The fiber space at $w_{\xi}$ is

$$
\mathcal{F}_{\xi}=\left\{\phi \in W^{2,2}\left(\Omega_{\xi}\right): \partial_{\nu} \phi=0 \text { on } \partial \Omega_{\xi}, \bar{\phi}=0, \phi \perp b_{j, \xi}^{k}, k=1, \ldots, K, j=1,2\right\}
$$

Also define

$$
\mathcal{E}_{\xi}=\left\{q \in L^{2}\left(\Omega_{\xi}\right): \bar{q}=0, q \perp b_{j, \xi}^{k}, k=1, \ldots, K, j=1,2\right\}
$$

and let $\pi_{\xi}$ be the projection to $\mathcal{E}_{\xi}$ as in the last section.

In the second step we solve the equation $\pi_{\xi} \circ S\left(w_{\xi}+\phi_{\xi}\right)=0$. First we must be able to invert the linearized operator $L_{\xi}$.

Lemma 6.5 The operator $\pi_{\xi} \circ L_{\xi}$ is one-to-one from $\mathcal{F}_{\xi}$ onto $\mathcal{E}_{\xi}$. There exists $C>0$ independent of $\epsilon$ such that $\|\phi\|_{W^{2,2}\left(\Omega_{\xi}\right)} \leqslant C \epsilon^{-4 / 3}\left\|\pi_{\xi} \circ L_{\xi}(\phi)\right\|_{L^{2}\left(\Omega_{\xi}\right)}$ for all $\phi \in \mathcal{F}_{\xi}$.

The proof of this lemma is given in Appendix F. Using a fixed point argument we obtain

Lemma 6.6 There exists $\phi_{\xi} \in \mathcal{F}_{\xi}$ so that $\pi_{\xi} \circ S_{\xi}\left(w_{\xi}+\phi_{\xi}\right)=0$. Moreover,

$$
\left\|\phi_{\xi}\right\|_{W^{2,2}\left(\Omega_{\xi}\right)}=O\left(e^{-(1+\delta) \sqrt{f^{\prime}(z)} \varphi(\xi) / \epsilon}\right)
$$

for some $\delta>0$ independent of $\epsilon$.

In the third and final step we maximize $I\left(w_{\xi}+\phi_{\xi}\right)$ with respect to $\xi$. Again it suffices to consider $I\left(w_{\xi}\right)$ based on the following lemma.

LEMMA 6.7

$$
I\left(w_{\xi}+\phi_{\xi}\right)=I\left(w_{\xi}\right)+O\left(e^{-(2+\delta) \sqrt{f^{\prime}(z)} \varphi(\xi) / \epsilon}\right)
$$

for some $\delta>0$ independent of $\epsilon$.

Combining Lemma 6.4 (2) and Lemma 6.7 we see that $I\left(w_{\xi}+\phi_{\xi}\right)$ has the asymptotic property

$$
C_{\epsilon}-C_{0} e^{a_{0} \epsilon^{-2 / 3}} e^{-2 \sqrt{f^{\prime}(z)} \varphi(\xi) / \epsilon} \leqslant I\left(w_{\xi}+\phi_{\xi}\right) \leqslant C_{\epsilon}-C_{1} e^{-a_{1} \epsilon^{-2 / 3}} e^{-2 \sqrt{f^{\prime}(z)} \varphi(\xi) / \epsilon} .
$$

As indicated at the beginning of this section $\varphi(\xi)$ has an interior maximum, so $I\left(w_{\xi}+\phi_{\xi}\right)$ is maximized at some $\xi_{*}$. It follows that $w_{\xi_{*}}+\phi_{\xi_{*}}$ is an exact solution of $S\left(w_{\xi_{*}}+\phi_{\xi_{*}}\right)=0$. This proves Theorem 6.1.

\section{Appendix A. Proof of Lemma 3.5}

Let us define

$$
p=H^{\prime}+\epsilon^{2 / 3} P^{\prime}-\overline{H^{\prime}+\epsilon^{2 / 3} P^{\prime}}
$$

where $H$ and $P$ are given in 2.3, 2.4.

Regarding the linear operator $L$ we have the following results. 
Lemma A.1 There exists a constant $C$ independent of $\epsilon$ such that $\|\psi\|_{\infty} \leqslant C\|L(\psi)\|_{\infty}$ for all $\psi \in \mathcal{F}$ with $\psi \perp p$.

Proof. Suppose that the lemma is false: there exist $\psi$ and some $r_{*}$ such that $\|\psi\|_{\infty}=\psi\left(r_{*}\right)=1$, $\psi \perp p$ and $L(\psi)=o(1)$. Then $r_{*}$ must lie in a neighborhood of $\rho$. The size of this neighborhood must be of order $\epsilon$. Otherwise $-\epsilon^{2} \Delta \psi\left(r_{*}\right) \geqslant 0, \overline{f^{\prime}(w) \psi}=\overline{\left(f^{\prime}(w)-f^{\prime}(z)\right) \psi}=o(1)($ as $\bar{\psi}=0)$, and $f^{\prime}\left(w\left(r_{*}\right)\right) \psi\left(r_{*}\right)$ is positive and bounded away from 0 independent of $\epsilon$. Then $L(\psi)=o(1)$ is not satisfied at $r_{*}$.

So let us assume that $r_{*}$ is in a neighborhood, of size $\epsilon$, of $\rho$. Then $\psi(\rho+\epsilon t) \rightarrow \Psi_{0}(t)$ in $C_{\text {loc }}^{2}(\mathbb{R})$ as $\epsilon$ tends to 0 . We have $-\Psi_{0}^{\prime \prime}+f^{\prime}(H) \Psi_{0}=0$. Therefore $\Psi_{0}=c H^{\prime}$ for some constant $c \neq 0$. On the other hand, $\psi \perp p$ implies

$$
0=\left\langle\psi, H^{\prime}-\overline{H^{\prime}}+\epsilon^{2 / 3}\left(P^{\prime}-\overline{P^{\prime}}\right)\right\rangle=2 \pi c \epsilon \rho \int_{\mathbb{R}}\left(H^{\prime}\right)^{2} \mathrm{~d} t+o\left(\epsilon^{4 / 3}\right)=2 \pi c \epsilon \rho \tau+o\left(\epsilon^{4 / 3}\right),
$$

which is possible only if $c=0$. A contradiction.

Before proving the estimate $\|\phi\|_{\infty} \leqslant C \epsilon^{-4 / 3}\|L(\phi)\|_{\infty}$ of Lemma 3.5, we note that the estimate implies that $L$ is one-to-one. The surjectivity of $L$ means that for any $q \in \mathcal{Y}$ there is $\phi \in \mathcal{F}$ so that $L(\phi)=q$. We write this equation as

$$
\epsilon^{2} \phi+(-\Delta)^{-1}\left(f^{\prime}(w) \phi-\overline{f^{\prime}(w) \phi}\right)=(-\Delta)^{-1} q
$$

where $(-\Delta)^{-1}$ is a bijection from $\mathcal{Y}$ onto $\mathcal{F}$. The left side of the equation defines an operator from $\mathcal{F}$ to itself which is of the form " $\epsilon$ identity + compact". For this operator, $\mathcal{F}$ is equipped with the $W^{2,2}$ norm. The Fredholm alternative asserts that the equation is solvable if and only if the homogeneous equation

$$
\epsilon^{2} \phi+(-\Delta)^{-1}\left(f^{\prime}(w) \phi-\overline{f^{\prime}(w) \phi}\right)=0
$$

only has the trivial solution. But this is a consequence of $L$ being one-to-one.

Hence it suffices to prove the estimate. Suppose it is not true. Then there exists $\phi$ with $\|\phi\|=1$ and $L(\phi)=o\left(\epsilon^{4 / 3}\right)$ along a sequence of $\epsilon$ that tends to 0 .

Decompose $\phi$ into

$$
\phi=c p+\phi^{\perp}, \quad p \perp \phi^{\perp} .
$$

We start with $L(p)$. First we estimate

$$
L\left(H^{\prime}-\overline{H^{\prime}}\right)=-\epsilon^{2}\left(H^{\prime}\right)_{r r}-\frac{\epsilon^{2}}{r}\left(H^{\prime}\right)_{r}+f^{\prime}(w)\left(H^{\prime}-\overline{H^{\prime}}\right)-\overline{f^{\prime}(w)\left(H^{\prime}-\overline{H^{\prime}}\right)},
$$

in which

$$
\begin{aligned}
\overline{f^{\prime}(w) H^{\prime}} & =2 \int_{0}^{1}\left(f^{\prime}(H)+\epsilon^{2 / 3} P f^{\prime \prime}(H)\right) H^{\prime} r \mathrm{~d} r+o\left(\epsilon^{4 / 3}\right) \\
& =2 \epsilon \int_{\mathbb{R}}\left[f^{\prime}(H) \rho+\epsilon t f^{\prime}(H)+\epsilon^{2 / 3} P f^{\prime \prime}(H) \rho\right] H^{\prime} \mathrm{d} t+o\left(\epsilon^{4 / 3}\right)=o\left(\epsilon^{4 / 3}\right),
\end{aligned}
$$

since $\int_{\mathbb{R}} f^{\prime}(H) H^{\prime} \mathrm{d} t=\int_{\mathbb{R}} t f^{\prime}(H) H^{\prime} \mathrm{d} t=\int_{\mathbb{R}} P f^{\prime \prime}(H) H^{\prime} \mathrm{d} t=0\left(t f^{\prime}(H) H^{\prime}\right.$ and $P f^{\prime \prime}(H) H^{\prime}$ are 
odd). Then

$$
\begin{aligned}
& L\left(H^{\prime}-\overline{H^{\prime}}\right) \\
& =\left(f^{\prime}(w)-f^{\prime}(H)\right) H^{\prime}-\frac{\epsilon}{r} H^{\prime \prime}+\left(\overline{f^{\prime}(w)}-f^{\prime}(w)\right) \overline{H^{\prime}}+o\left(\epsilon^{4 / 3}\right) \\
& =\epsilon^{2 / 3} f^{\prime \prime}(H) P H^{\prime}+\epsilon^{4 / 3}\left(f^{\prime \prime}(H) Q+\frac{f^{\prime \prime \prime}(H) P^{2}}{2}\right) H^{\prime}-\frac{\epsilon}{r} H^{\prime \prime}+\left(\overline{f^{\prime}(w)}-f^{\prime}(w)\right) \overline{H^{\prime}}+o\left(\epsilon^{4 / 3}\right) .
\end{aligned}
$$

By differentiating [2.4 we have

$$
-P^{\prime \prime \prime}+f^{\prime}(H) P^{\prime}+f^{\prime \prime}(H) H^{\prime} P-\frac{\epsilon^{1 / 3}}{\rho} H^{\prime \prime}=0 .
$$

Then

$$
\begin{aligned}
& L\left(P^{\prime}-\overline{P^{\prime}}\right)=-\epsilon^{2}\left(P^{\prime}\right)_{r r}-\frac{\epsilon^{2}}{r}\left(P^{\prime}\right)_{r}+f^{\prime}(w)\left(P^{\prime}-\overline{P^{\prime}}\right)-\overline{f^{\prime}(w)\left(P^{\prime}-\overline{P^{\prime}}\right)} \\
& =\left(f^{\prime}(w)-f^{\prime}(H)\right) P^{\prime}-f^{\prime \prime}(H) H^{\prime} P+\frac{\epsilon^{1 / 3}}{\rho} H^{\prime \prime}-\frac{\epsilon}{r} P^{\prime \prime}+\left(\overline{f^{\prime}(w)}-f^{\prime}(w)\right) \overline{P^{\prime}}+o\left(\epsilon^{2 / 3}\right) \\
& =\epsilon^{2 / 3} f^{\prime \prime}(H) P P^{\prime}-f^{\prime \prime}(H) H^{\prime} P+\frac{\epsilon^{1 / 3}}{\rho} H^{\prime \prime}-\frac{\epsilon}{r} P^{\prime \prime}+\left(\overline{f^{\prime}(w)}-f^{\prime}(w)\right) \overline{P^{\prime}}+o\left(\epsilon^{2 / 3}\right),
\end{aligned}
$$

where we have used the fact that

$$
\overline{f^{\prime}(w) P^{\prime}}=2 \int_{0}^{1} f^{\prime}(w) P^{\prime} r \mathrm{~d} r=o\left(\epsilon^{2 / 3}\right) .
$$

Therefore

$$
\begin{aligned}
L(p)= & \epsilon^{4 / 3}\left[\left(f^{\prime \prime}(H) Q+\frac{f^{\prime \prime \prime}(H) P^{2}}{2}\right) H^{\prime}+f^{\prime \prime}(H) P P^{\prime}+\left(\frac{1}{\epsilon^{1 / 3} \rho}-\frac{1}{\epsilon^{1 / 3} r}\right) H^{\prime \prime}-\frac{\epsilon^{1 / 3}}{r} P^{\prime \prime}\right] \\
& +\left(\overline{f^{\prime}(w)}-f^{\prime}(w)\right) \overline{H^{\prime}+\epsilon^{2 / 3} P^{\prime}}+o\left(\epsilon^{4 / 3}\right) .
\end{aligned}
$$

On the other hand,

$$
\begin{aligned}
\overline{H^{\prime}} & =2 \int_{0}^{1} H^{\prime} r \mathrm{~d} r=2 \epsilon \int_{\mathbb{R}} H^{\prime}(t)(\rho+\epsilon t) \mathrm{d} t+o\left(\epsilon^{4 / 3}\right) \\
& =2 \epsilon \rho \int_{\mathbb{R}} H^{\prime}(t) \mathrm{d} t+2 \epsilon^{2} \int_{\mathbb{R}} H^{\prime}(t) t \mathrm{~d} t+o\left(\epsilon^{4 / 3}\right)=-2 \epsilon \rho+o\left(\epsilon^{4 / 3}\right)
\end{aligned}
$$

since $H^{\prime}(t) t$ is odd, and

$$
\overline{P^{\prime}}=2 \int_{0}^{1} P^{\prime} r \mathrm{~d} r=2 \epsilon \rho \int_{\mathbb{R}} P^{\prime} \mathrm{d} t+O\left(\epsilon^{2}\right)=O\left(\epsilon^{2}\right)
$$

since $P^{\prime}$ is odd. We find

$$
\overline{H^{\prime}+\epsilon^{2 / 3} P^{\prime}}=-2 \epsilon \rho+o\left(\epsilon^{4 / 3}\right) .
$$


Hence we deduce that

$$
\begin{aligned}
L(p)= & \epsilon^{4 / 3}\left[\left(f^{\prime \prime}(H) Q+\frac{f^{\prime \prime \prime}(H) P^{2}}{2}\right) H^{\prime}+f^{\prime \prime}(H) P P^{\prime}+\left(\frac{1}{\epsilon^{1 / 3} \rho}-\frac{1}{\epsilon^{1 / 3} r}\right) H^{\prime \prime}-\frac{\epsilon^{1 / 3}}{r} P^{\prime \prime}\right] \\
& -2 \epsilon \rho\left(\overline{f^{\prime}(w)}-f^{\prime}(w)\right)+o\left(\epsilon^{4 / 3}\right) .
\end{aligned}
$$

Note that in A.4,

$$
\left(\frac{1}{\epsilon^{1 / 3} \rho}-\frac{1}{\epsilon^{1 / 3} r}\right) H^{\prime \prime}=\frac{\epsilon^{2 / 3} t H^{\prime \prime}(t)}{\rho(\rho+\epsilon t)}=O(1) .
$$

In particular

$$
L(p)=O\left(\epsilon^{4 / 3}\right)
$$

We write $L(\phi)=o\left(\epsilon^{4 / 3}\right)$ as

$$
L\left(\phi^{\perp}\right)=-c L(p)+o\left(\epsilon^{4 / 3}\right)=O\left(\epsilon^{4 / 3}|c|\right)+o\left(\epsilon^{4 / 3}\right) .
$$

We deduce from the last equation and Lemma A.1 that

$$
\phi^{\perp}=O\left(\epsilon^{4 / 3}|c|\right)+o\left(\epsilon^{4 / 3}\right) .
$$

We now return to

$$
c L(p)+L\left(\phi^{\perp}\right)=o\left(\epsilon^{4 / 3}\right) .
$$

Multiply the equation by $p$ and integrate over $\Omega$ to deduce

$$
c\langle L(p), p\rangle+\left\langle\phi^{\perp}, L(p)\right\rangle=o\left(\epsilon^{8 / 3}\right) .
$$

Note that we have used the fact $\|p\|_{1}=O\left(\epsilon^{4 / 3}\right)$ to obtain the right side of A.7.

The two terms on the left side are calculated as follows:

$$
\langle L(p), p\rangle=c_{0} \epsilon^{8 / 3}+o\left(\epsilon^{8 / 3}\right), \quad c_{0} \neq 0,
$$

where $c_{0}$ is independent of $\epsilon$. To see this we note that $P^{\prime}$ decays exponentially fast. Then A.4 implies that

$$
\begin{aligned}
&\langle L(p), p\rangle=\left\langle L\left(H^{\prime}-\overline{H^{\prime}}+\epsilon^{2 / 3}\left(P^{\prime}-\overline{P^{\prime}}\right)\right), H^{\prime}+\epsilon^{2 / 3} P^{\prime}\right\rangle \\
&= \epsilon^{4 / 3}\left\langle\left(f^{\prime \prime}(H) Q+\frac{f^{\prime \prime \prime}(H) P^{2}}{2}\right) H^{\prime}+f^{\prime \prime}(H) P P^{\prime}+\frac{\epsilon^{2 / 3} t H_{j}^{\prime \prime}}{\rho r}-\frac{\epsilon^{1 / 3}}{r} P^{\prime \prime}, H^{\prime}+\epsilon^{2 / 3} P^{\prime}\right\rangle \\
&-2 \epsilon \rho\left\langle\overline{f^{\prime}(w)}-f^{\prime}(w), H^{\prime}+\epsilon^{2 / 3} P^{\prime}\right\rangle+o\left(\epsilon^{8 / 3}\right) \\
&= \epsilon^{4 / 3}\left\langle\left(f^{\prime \prime}(H) Q+\frac{f^{\prime \prime \prime}(H) P^{2}}{2}\right) H^{\prime}+f^{\prime \prime}(H) P P^{\prime}+\frac{\epsilon^{2 / 3} t H_{j}^{\prime \prime}}{\rho r}-\frac{\epsilon^{1 / 3}}{r} P^{\prime \prime}, H^{\prime}\right\rangle \\
&-2 \epsilon \rho\left\langle\overline{f^{\prime}(w)}-f^{\prime}(w), H^{\prime}\right\rangle+o\left(\epsilon^{8 / 3}\right) \\
&= 2 \pi \epsilon^{7 / 3} \rho\left\{\int_{\mathbb{R}}\left[\left(f^{\prime \prime}(H) Q+\frac{f^{\prime \prime \prime}(H) P^{2}}{2}\right) H^{\prime}+f^{\prime \prime}(H) P P^{\prime}+\frac{\epsilon^{2 / 3} t H^{\prime \prime}}{\rho^{2}}-\frac{\epsilon^{1 / 3}}{\rho} P^{\prime \prime}\right] H^{\prime} \mathrm{d} t\right\} \\
&+4 \epsilon^{2} \pi \rho^{2} \overline{f^{\prime}(w)}+o\left(\epsilon^{8 / 3}\right) .
\end{aligned}
$$


To find the integral in A.8, we differentiate 2.5$]$ to obtain

$-Q^{\prime \prime \prime}+f^{\prime}(H) Q^{\prime}+f^{\prime \prime}(H) H^{\prime} Q-\frac{\epsilon^{1 / 3}}{\rho} P^{\prime \prime}+\frac{\epsilon^{2 / 3}}{\rho^{2}}\left(H^{\prime}+t H^{\prime \prime}\right)+\frac{f^{\prime \prime \prime}(H) H^{\prime} P^{2}}{2}+f^{\prime \prime}(H) P P^{\prime}=0$.

Multiplying by $H^{\prime}$ and integrating over $(-\infty, \infty)$ yield

$$
\int_{\mathbb{R}}\left[f^{\prime \prime}(H) Q\left(H^{\prime}\right)^{2}-\frac{\epsilon^{1 / 3} P^{\prime \prime} H^{\prime}}{\rho}+\frac{\epsilon^{2 / 3}}{\rho^{2}}\left(\left(H^{\prime}\right)^{2}+t H^{\prime \prime} H^{\prime}\right)+\frac{f^{\prime \prime \prime}(H) P^{2}\left(H^{\prime}\right)^{2}}{2}+f^{\prime \prime}(H) P P^{\prime} H^{\prime}\right] \mathrm{d} t=0 .
$$

The integral in A.8) now becomes

$$
-\frac{\epsilon^{2 / 3}}{\rho^{2}} \int_{\mathbb{R}}\left(H^{\prime}\right)^{2} \mathrm{~d} t=-\frac{\epsilon^{2 / 3} \tau}{\rho^{2}} .
$$

Therefore

$$
\langle L p, p\rangle=4 \pi \epsilon^{2} \rho^{2} f^{\prime}(0)-\frac{2 \pi \epsilon^{3} \tau}{\rho}+o\left(\epsilon^{8 / 3}\right) .
$$

For the smaller droplet solution with $\rho_{0}<\left(\tau /\left(2 f^{\prime}(0)\right)\right)^{1 / 3}$, the first two terms on the right hand side of A.9 give a negative number of order $\epsilon^{8 / 3}$. For the larger droplet solution with $\rho_{0}>\left(\tau /\left(2 f^{\prime}(0)\right)\right)^{1 / 3}$, the first two terms on the right hand side of A.9 give a positive number of order $\epsilon^{8 / 3}$. In each of the two cases the right side is $c_{0} \epsilon^{8 / 3}+o\left(\epsilon^{8 / 3}\right)$ for some $c_{0} \neq 0$.

Next we estimate $\left\langle\phi^{\perp}, L(p)\right\rangle$. Although, by A.5 and A.6, we could have

$$
\left\langle\phi^{\perp}, L(p)\right\rangle=\left(O\left(\epsilon^{4 / 3}|c|\right)+o\left(\epsilon^{4 / 3}\right)\right) O\left(\epsilon^{4 / 3}\right),
$$

this estimate is not good enough. Instead we note that

$$
\left|\left\langle\phi^{\perp}, L(p)\right\rangle\right| \leqslant\left\|\phi^{\perp}\right\|_{\infty}\|L(p)\|_{1}=\left(O\left(\epsilon^{4 / 3}|c|\right)+o\left(\epsilon^{4 / 3}\right)\right)\|L(p)\|_{1} .
$$

Although $L(p)=O\left(\epsilon^{4 / 3}\right)$, a close observation of A.4 shows that only in a neighborhood, whose size is of order $\epsilon, L(p)$ is of order $O\left(\epsilon^{4 / 3}\right)$ and outside of this neighborhood $L(p)$ is of order $o\left(\epsilon^{4 / 3}\right)$. Therefore

$$
\|L(p)\|_{1}=o\left(\epsilon^{4 / 3}\right)
$$

and consequently

$$
\left\langle\phi^{\perp}, L(p)\right\rangle=\left(O\left(\epsilon^{4 / 3}|c|\right)+o\left(\epsilon^{4 / 3}\right)\right) o\left(\epsilon^{4 / 3}\right) .
$$

Then A.77 becomes

$$
c\left(c_{0} \epsilon^{8 / 3}+o\left(\epsilon^{8 / 3}\right)\right)+\left(O\left(\epsilon^{4 / 3}|c|\right)+o\left(\epsilon^{4 / 3}\right)\right) o\left(\epsilon^{4 / 3}\right)=o\left(\epsilon^{8 / 3}\right),
$$

which implies that

$$
c=o(1)
$$

By A.6 we find that $\phi^{\perp}=o\left(\epsilon^{4 / 3}\right)$ and $\phi=o(1)$. This is a contradiction to the assumption that $\|\phi\|_{\infty}=1$. 


\section{Appendix B. Proof of Theorem 4.1}

Let $\lambda$ be an eigenvalue. We claim that

$$
\liminf _{\epsilon \rightarrow 0} \lambda \geqslant 0
$$

This may be proved by the maximum principle argument as in the proof of Lemma A.1.

We now only need to consider $\lambda$ that satisfies

$$
\lim _{\epsilon \rightarrow 0} \lambda=0
$$

Such an eigenvalue is called a critical eigenvalue. Let $\phi$ be an eigenfunction corresponding to $\lambda$. We decompose $\phi$ into

$$
\phi=c p+\phi^{\perp}, \quad p \perp \phi^{\perp},
$$

where $p$ is given by the same formula A.1. We write $L(\phi)=\lambda \phi$ as

$$
c L(p)+L\left(\phi^{\perp}\right)=\lambda\left(c p+\phi^{\perp}\right) .
$$

Since $L(p)=O\left(\epsilon^{4 / 3}\right)$, we find

$$
L\left(\phi^{\perp}\right)=O\left(\epsilon^{4 / 3}|c|\right)+O(|\lambda|)\left(|c|+\left\|\phi^{\perp}\right\|_{\infty}\right) .
$$

As in Lemma A.1 we deduce that

$$
\left\|\phi^{\perp}\right\|_{\infty}=O\left(\epsilon^{4 / 3}|c|\right)+O(|\lambda|)\left(|c|+\left\|\phi^{\perp}\right\|_{\infty}\right),
$$

which implies, since $\lambda=o(1)$,

$$
\left\|\phi^{\perp}\right\|_{\infty}=O\left(\epsilon^{4 / 3}|c|\right)+O(|\lambda|)|c| .
$$

We multiply $\mathrm{B} .1$ by $p$ and integrate to find

$$
c\langle L(p), p\rangle+\left\langle\phi^{\perp}, L(p)\right\rangle=\lambda c\|p\|_{2}^{2} .
$$

The right hand side is

$$
\lambda c\left(2 \pi \epsilon \rho \tau+o\left(\epsilon^{4 / 3}\right)\right) .
$$

We estimate the second term on the left:

$$
\left|\left\langle\phi^{\perp}, L(p)\right\rangle\right| \leqslant\left\|\phi^{\perp}\right\|_{\infty}\|L(p)\|_{1} .
$$

Here $\left\|\phi^{\perp}\right\|_{\infty}$ is given in B.2. By A.10 we find

$$
\left\langle\phi^{\perp}, L(p)\right\rangle=\left(O\left(\epsilon^{4 / 3}|c|\right)+O(|\lambda|)|c|\right) o\left(\epsilon^{4 / 3}\right) .
$$

We now return to B.3 and, with the help of A.9, we find

$c\left(4 \pi \epsilon^{2} \rho^{2} f^{\prime}(0)-\frac{2 \pi \epsilon^{3} \tau}{\rho}+o\left(\epsilon^{8 / 3}\right)\right)+\left(O\left(\epsilon^{4 / 3}|c|\right)+O(|\lambda|)|c|\right) o\left(\epsilon^{4 / 3}\right)=\lambda c\left(2 \pi \epsilon \rho \tau+o\left(\epsilon^{4 / 3}\right)\right)$. 
Ignoring the higher order terms, we obtain

$$
\lim _{\epsilon \rightarrow 0} \epsilon^{-4 / 3} \lambda=\frac{2 f^{\prime}(0) \rho_{0}}{\tau}-\frac{1}{\rho_{0}^{2}} .
$$

This gives the asymptotic expansion of $\lambda$, claimed in the theorem. Knowing $\lambda=O\left(\epsilon^{4 / 3}\right)$, we return to $B .2$ to deduce

$$
\phi=c p+O\left(\epsilon^{4 / 3}|c|\right),
$$

which gives the expansion of the eigenfunction.

Recall that the smaller droplet solution has $\rho_{0}<\left(\tau /\left(2 f^{\prime}(0)\right)\right)^{1 / 3}$ and the larger droplet solution has $\rho_{0}>\left(\tau /\left(2 f^{\prime}(0)\right)\right)^{1 / 3}$. The right hand side of $\mathrm{B} .4$ is negative if $\rho_{0}<\left(\tau /\left(2 f^{\prime}(0)\right)\right)^{1 / 3}$, and positive if $\rho_{0}>\left(\tau /\left(2 f^{\prime}(0)\right)\right)^{1 / 3}$. Hence the solution with smaller $\rho_{0}$ leads to a negative $\lambda$ and the one with larger $\rho_{0}$ leads to a positive $\lambda$. Therefore the smaller droplet solution is unstable in the radial class and the larger droplet solution is stable in the radial class.

The critical eigenvalue $\lambda$ is unique. Otherwise there would be two eigenfunctions $\phi_{1}$ and $\phi_{2}$ with the same expansion property, i.e. $c p+O\left(\epsilon^{4 / 3}|c|\right)$. On the other hand, by the self-adjointness of $L$, $\phi_{1}$ and $\phi_{2}$ must be perpendicular. One can then find a contradiction (see [16, Section 4]).

It can also be shown, as in [16, Section 4], that there always exists a simple eigenvalue with the property claimed in the theorem.

\section{Appendix C. Proof of Lemma 5.4}

We start with an estimate of $\eta_{\xi}$. Let $\tilde{v}_{\xi}=v(\cdot-\xi)-z$ and $\tilde{w}_{\xi}=w_{\xi}-z-\eta_{\xi}=\tilde{v}_{\xi}+g_{\xi}$. Then $\tilde{w}_{\xi}$ satisfies the equation

$$
-\epsilon^{2} \Delta \tilde{w}_{\xi}+f^{\prime}(z) \tilde{w}_{\xi}+h\left(\tilde{v}_{\xi}\right)=0, \quad \partial_{\nu} \tilde{w}_{\xi}=0 \quad \text { on } \partial \Omega .
$$

Integrate the above equation to deduce

$$
f^{\prime}(z)\left(m-z-\eta_{\xi}\right)|\Omega|+\int_{\Omega} h\left(\tilde{v}_{\xi}\right) \mathrm{d} x=0 .
$$

From $(5.8)$ we deduce

$$
\eta_{\xi}=-\frac{1}{f^{\prime}(z)|\Omega|} \int_{\mathbb{R}^{2} \backslash \Omega} h\left(\tilde{v}_{\xi}\right) \mathrm{d} x .
$$

If we multiply the equation for $\tilde{v}_{\xi}$ by $\tilde{v}_{\xi}$ and integrate over $\mathbb{R}^{2} \backslash \Omega$, we find

$$
\begin{aligned}
\int_{\mathbb{R}^{2} \backslash \Omega}\left[\epsilon^{2}\left|\nabla \tilde{v}_{\xi}\right|^{2}+f^{\prime}(z) \tilde{v}_{\xi}^{2}\right] \mathrm{d} x & =-\epsilon^{2} \int_{\partial \Omega} \frac{\partial \tilde{v}_{\xi}}{\partial v} \tilde{v}_{\xi} \mathrm{d} s-\int_{\mathbb{R}^{2} \backslash \Omega} h\left(\tilde{v}_{\xi}\right) \tilde{v}_{\xi} \mathrm{d} x \\
& =-\epsilon^{2} \int_{\partial \Omega} \frac{\partial \tilde{v}_{\xi}}{\partial \nu} \tilde{v}_{\xi} \mathrm{d} s+O\left(e^{-(2+\delta) \sqrt{f^{\prime}(z)} d_{\xi} / \epsilon}\right)
\end{aligned}
$$

for some $\delta>0$. Here we have used the fact that $h\left(\tilde{v}_{\xi}\right)=O\left(\tilde{v}_{\xi}^{2}\right)$ and Lemma 2.1. In the boundary integral, $v$ points outwards from $\Omega$ (into $\mathbb{R}^{2} \backslash \Omega$ ). Consequently,

$$
\int_{\mathbb{R}^{2} \backslash \Omega}\left|h\left(\tilde{v}_{\xi}\right)\right| \mathrm{d} x \leqslant C \int_{\mathbb{R}^{2} \backslash \Omega} \tilde{v}_{\xi}^{2} \mathrm{~d} x \leqslant-C \epsilon^{2} \int_{\partial \Omega} \frac{\partial \tilde{v}_{\xi}}{\partial \nu} \tilde{v}_{\xi} \mathrm{d} s+O\left(e^{-(2+\delta) \sqrt{f^{\prime}(z)}} d_{\xi} / \epsilon\right) .
$$


So we have

$$
\left|\eta_{\xi}\right| \leqslant-C \epsilon^{2} \int_{\partial \Omega} \frac{\partial \tilde{v}_{\xi}}{\partial v} \tilde{v}_{\xi} \mathrm{d} s+O\left(e^{-(2+\delta) \sqrt{f^{\prime}(z)} d_{\xi} / \epsilon}\right) .
$$

If we apply Lemma 2.1 , then we obtain

$$
\eta_{\xi}=O\left(e^{-2 \sqrt{f^{\prime}(z)} d \xi / \epsilon}\right) .
$$

Now we turn our attention to $I\left(w_{\xi}\right)$ to see how it depends on $\xi$. Here

$$
\begin{aligned}
I\left(w_{\xi}\right) & =\int_{\Omega}\left[\frac{\epsilon^{2}\left|\nabla \tilde{w}_{\xi}\right|^{2}}{2}+F\left(\tilde{w}_{\xi}+z+\eta_{\xi}\right)\right] \mathrm{d} x \\
& =\int_{\Omega}\left[\frac{\epsilon^{2}\left|\nabla \tilde{w}_{\xi}\right|^{2}}{2}+F\left(z+\tilde{w}_{\xi}\right)+f\left(z+\tilde{w}_{\xi}\right) \eta_{\xi}\right] \mathrm{d} x+O\left(\eta_{\xi}^{2}\right) \\
& =\int_{\Omega}\left[\frac{\epsilon^{2}\left|\nabla \tilde{w}_{\xi}\right|^{2}}{2}+F\left(z+\tilde{w}_{\xi}\right)\right] \mathrm{d} x+O\left(\epsilon^{2 / 3} \eta_{\xi}\right) \\
& =\int_{\Omega}\left[\frac{\epsilon^{2}\left|\nabla \tilde{w}_{\xi}\right|^{2}}{2}+F(z)+f(z) \tilde{w}_{\xi}+\frac{f^{\prime}(z)}{2} \tilde{w}_{\xi}^{2}+H\left(\tilde{w}_{\xi}\right)\right] \mathrm{d} x+O\left(\epsilon^{2 / 3} \eta_{\xi}\right) \\
& =\int_{\Omega}\left[\frac{\epsilon^{2}\left|\nabla \tilde{w}_{\xi}\right|^{2}}{2}+\frac{f^{\prime}(z)}{2} \tilde{w}_{\xi}^{2}+H\left(\tilde{w}_{\xi}\right)\right] \mathrm{d} x+|\Omega|\left(F(z)+f(z)\left(m-z-\eta_{\xi}\right)\right)+O\left(\epsilon^{2 / 3} \eta_{\xi}\right) \\
& =\tilde{I}\left(\tilde{w}_{\xi}\right)+|\Omega|(F(z)+f(z)(m-z))+O\left(\epsilon^{2 / 3} \eta_{\xi}\right)
\end{aligned}
$$

where the second term in the last line is independent of $\xi$ and

$$
\tilde{I}\left(\tilde{w}_{\xi}\right)=\int_{\Omega}\left[\frac{\epsilon^{2}\left|\nabla \tilde{w}_{\xi}\right|^{2}}{2}+\frac{f^{\prime}(z)}{2} \tilde{w}_{\xi}^{2}+H\left(\tilde{w}_{\xi}\right)\right] \mathrm{d} x .
$$

To compute $\tilde{I}\left(\tilde{w}_{\xi}\right)$ we use the integral identity

$$
\int_{\Omega}\left[\epsilon^{2}\left|\nabla \tilde{w}_{\xi}\right|^{2}+f^{\prime}(z) \tilde{w}_{\xi}^{2}+h\left(\tilde{v}_{\xi}\right) \tilde{w}_{\xi}\right] \mathrm{d} x=0,
$$

which follows from the equation for $\tilde{w}_{\xi}$. We can rewrite $\tilde{I}\left(\tilde{w}_{\xi}\right)$ as

$$
\begin{aligned}
\tilde{I}\left(\tilde{w}_{\xi}\right) & =\int_{\Omega}\left[H\left(\tilde{w}_{\xi}\right)-\frac{1}{2} h\left(\tilde{v}_{\xi}\right) \tilde{w}_{\xi}\right] \mathrm{d} x \\
& =\int_{\Omega}\left[H\left(\tilde{v}_{\xi}\right)-\frac{1}{2} h\left(\tilde{v}_{\xi}\right) \tilde{v}_{\xi}\right] \mathrm{d} x+\frac{1}{2} \int_{\Omega} h\left(\tilde{v}_{\xi}\right) g_{\xi} \mathrm{d} x+O\left(\int_{\Omega} \tilde{v}_{\xi} g_{\xi}^{2} \mathrm{~d} x\right) .
\end{aligned}
$$

The three terms are estimated as follows. The first is

$$
\begin{aligned}
\int_{\Omega}\left[H\left(\tilde{v}_{\xi}\right)-\frac{1}{2} h\left(\tilde{v}_{\xi}\right) \tilde{v}_{\xi}\right] \mathrm{d} x & =\int_{\mathbb{R}^{2}}\left[H(\tilde{v})-\frac{1}{2} h(\tilde{v}) \tilde{v}\right] \mathrm{d} x+\int_{\mathbb{R}^{2} \backslash \Omega}\left[H\left(\tilde{v}_{\xi}\right)-\frac{1}{2} h\left(\tilde{v}_{\xi}\right) \tilde{v}_{\xi}\right] \mathrm{d} x \\
& =\int_{\mathbb{R}^{2}}\left[H(\tilde{v})-\frac{1}{2} h(\tilde{v}) \tilde{v}\right] \mathrm{d} x+O\left(e^{-(2+\delta) \sqrt{f^{\prime}(z)} d_{\xi} / \epsilon}\right)
\end{aligned}
$$

for some $\delta>0$ by Lemma 2.1. Note that the integral in the last line is independent of $\xi$. 
Before we estimate the second term in C.4 we need to know a bit more about $g_{\xi}$. Let $\hat{g}$ be the solution of

$$
-\epsilon^{2} \Delta \hat{g}+f^{\prime}(z) \hat{g}=0, \quad \partial_{\nu} \hat{g}=1 \quad \text { on } \partial \Omega .
$$

Lemma C.1 (1) There exist $C>0$ and $a>0$ so that

$$
\left|g_{\xi}(x)\right| \leqslant C e^{a \epsilon^{-2 / 3}} e^{-\sqrt{f^{\prime}(z)} d_{\xi} / \epsilon} \hat{g}(x) .
$$

(2) There exist $C>0$ and $\delta>0$ such that

$$
g_{\xi}(x) \geqslant-C e^{-(1+\delta) \sqrt{f^{\prime}(z)} d_{\xi} / \epsilon} \hat{g}(x) .
$$

Proof. Note that on $\partial \Omega$,

$$
\partial_{\nu} g_{\xi}(x)=-v^{\prime}(|x-\xi|) \frac{\langle x-\xi, \nu\rangle}{|x-\xi|} .
$$

Item (1) follows from Lemma 2.1 and the comparison principle.

Fix $\delta$ small. Then for $x \in B_{(1+2 \delta) d}(\xi) \cap \partial \Omega$, we have $\langle x-\xi, v\rangle /|x-\xi|=1+O(\delta)$ and hence $\partial_{\nu} g_{\xi}(x)>0$ there. For $x \in \partial \Omega \backslash B_{(1+2 \delta) d_{\xi}}(\xi)$, we have, again by Lemma 2.1.

$$
\partial_{\nu} g_{\xi}(x) \geqslant-C e^{-(1+\delta) \sqrt{f^{\prime}(z)} d_{\xi} / \epsilon}
$$

By the comparison principle, we have (2).

Now we can estimate

$$
\begin{aligned}
\int_{\Omega} h\left(\tilde{v}_{\xi}\right) g_{\xi} \mathrm{d} x & =\int_{\Omega}\left(\epsilon^{2} \Delta \tilde{v}_{\xi}-f^{\prime}(z) \tilde{v}_{\xi}\right) g_{\xi} \mathrm{d} x=\epsilon^{2} \int_{\partial \Omega}\left[\frac{\partial \tilde{v}_{\xi}}{\partial v} g_{\xi}-\frac{\partial g_{\xi}}{\partial v} \tilde{v}_{\xi}\right] \mathrm{d} s \\
& =\epsilon^{2} \int_{\partial \Omega} \frac{\partial \tilde{v}_{\xi}}{\partial v}\left(g_{\xi}+\tilde{v}_{\xi}\right) \mathrm{d} x \\
& =\epsilon^{2} \int_{\partial \Omega \cap B_{(1+2 \delta) d_{\xi}}(\xi)} v^{\prime}(|x-\xi|) \frac{\langle x-\xi, v\rangle}{|x-\xi|}\left(\tilde{v}_{\xi}+g_{\xi}\right) \mathrm{d} s+O\left(e^{-(2+\delta) \sqrt{f^{\prime}(z)} d_{\xi} / \epsilon}\right)
\end{aligned}
$$

for some $\delta>0$ by Lemmas 2.1 and C.1.1).

Note that since $\langle x-\xi, \nu\rangle /|x-\xi|=1+O(\delta)$ for $x$ in $\partial \Omega \cap B_{(1+2 \delta)} d_{\xi}(\xi)$, for some positive $C$ and $a$ we have

$$
\begin{aligned}
\int_{\partial \Omega \cap B_{(1+2 \delta) d_{\xi}}(\xi)} \epsilon^{2} v^{\prime}(|x-\xi|) \frac{\langle x-\xi, v\rangle}{|x-\xi|} \tilde{v}_{\xi} & \leqslant-C e^{-a \epsilon^{-2 / 3}} \int_{\partial \Omega \cap B_{(1+2 \delta) d_{\xi}}(\xi)} e^{-2 \sqrt{f^{\prime}(z)}|x-\xi| / \epsilon} \\
& \leqslant-C e^{-a \epsilon^{-2 / 3}} e^{-2 \sqrt{f^{\prime}(z)} d_{\xi} / \epsilon}
\end{aligned}
$$

by Lemma2.1. By Lemma C.12),

$$
\int_{\partial \Omega \cap B_{(1+2 \delta) d}(\xi)} \epsilon^{2} v^{\prime}(|x-\xi|) \frac{\langle x-\xi, v\rangle}{|x-\xi|} g_{\xi} \mathrm{d} s \leqslant C e^{-(2+\delta) \sqrt{f^{\prime}(z)} d_{\xi} / \epsilon} .
$$

Thus we obtain

$$
\int_{\Omega} h\left(\tilde{v}_{\xi}\right) g_{\xi} \mathrm{d} x \leqslant-C e^{-a \epsilon^{-2 / 3}} e^{-2 \sqrt{f^{\prime}(z)} d_{\xi} / \epsilon} .
$$


On the other hand, by Lemmas 2.1 and C.11), we have

$$
\left|\int_{\Omega} h\left(\tilde{v}_{\xi}\right) g_{\xi} \mathrm{d} x\right| \leqslant C e^{a \epsilon^{-2 / 3}} e^{-2 \sqrt{f^{\prime}(z)} d_{\xi} / \epsilon} .
$$

Combining the last two, we have the following important estimate:

$$
-C_{1} e^{a_{1} \epsilon^{-2 / 3}} e^{-2 \sqrt{f^{\prime}(z)} d_{\xi} / \epsilon} \leqslant \int_{\Omega} h\left(\tilde{v}_{\xi}\right) g_{\xi} \mathrm{d} x \leqslant-C_{0} e^{-a_{0} \epsilon^{-2 / 3}} e^{-2 \sqrt{f^{\prime}(z)} d_{\xi} / \epsilon} .
$$

To estimate the third term in (C.4) we let $\iota$ be a small positive number and divide $\Omega$ into $\Omega_{1}$ which consists of points in $\Omega$ whose distance to $\partial \Omega$ is less than $\iota$, and $\Omega_{2}=\Omega \backslash \Omega_{1}$. On $\Omega_{1}$, since $g_{\xi}=O\left(e^{-\sqrt{f^{\prime}(z)} d_{\xi} / \epsilon}\right)$ and $\tilde{v}_{\xi}$ is exponentially small by Lemma 2.1 , we have

$$
\int_{\Omega_{1}} \tilde{v}_{\xi} g_{\xi}^{2} \mathrm{~d} x=O\left(e^{-(2+\delta) \sqrt{f^{\prime}(z)} d_{\xi} / \epsilon}\right)
$$

for some $\delta>0$. On $\Omega_{2}$ we know that $\hat{g}=O\left(e^{-\delta_{1} / \epsilon}\right)$ for some $\delta_{1}>0$, and by Lemma C.1. $g_{\xi}(x)=\hat{g}(x) O\left(e^{a \epsilon^{-2 / 3}} e^{-\sqrt{f^{\prime}(z)} d_{\xi} / \epsilon}\right)$. Again we have

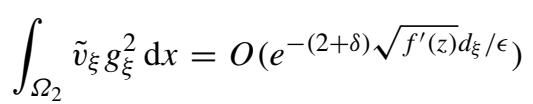

for some $\delta>0$. So on the whole $\Omega$ we have

$$
\int_{\Omega} \tilde{v}_{\xi} g_{\xi}^{2} \mathrm{~d} x=O\left(e^{-(2+\delta) \sqrt{f^{\prime}(z)} d \xi / \epsilon}\right) \quad \text { for some } \delta>0 .
$$

Before we can prove Lemma 5.4 (2) by combining C.5 C.7), we must deal with the $O\left(\epsilon^{2 / 3} \eta_{\xi}\right)$ term in (C.3). Fortunately (C.1) and the estimate of $\int_{\Omega} h\left(\tilde{v}_{\xi}\right) g_{\xi} \mathrm{d} x$ imply that

$$
O\left(\epsilon^{2 / 3} \eta_{\xi}\right)+\frac{1}{2} \int_{\Omega} h\left(\tilde{v}_{\xi}\right) g_{\xi} \mathrm{d} x=\left(\frac{1}{2}+O\left(\epsilon^{2 / 3}\right)\right) \int_{\Omega} h\left(\tilde{v}_{\xi}\right) g_{\xi} \mathrm{d} x .
$$

Lemma 5.4(2) now follows from (C.3 C.77.

To show Lemma 5.4(1), note that

$$
-\epsilon^{2} \Delta w_{\xi}+f\left(w_{\xi}\right)=\beta+f^{\prime}(z) \eta_{\xi}+h\left(\tilde{v}_{\xi}+g_{\xi}+\eta_{\xi}\right)-h\left(\tilde{v}_{\xi}\right) .
$$

We focus on

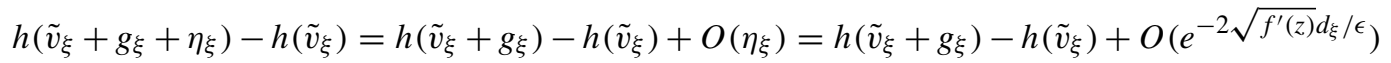

by (C.2). We then argue as in the proof of Lemma 3.4 to conclude that

$$
h\left(\tilde{v}_{\xi}+g_{\xi}\right)-h\left(\tilde{v}_{\xi}\right)=O\left(e^{-(1+\delta) \sqrt{f^{\prime}(z)} d_{\xi} / \epsilon}\right) .
$$

This shows that

$$
-\epsilon^{2} \Delta w_{\xi}+f\left(w_{\xi}\right)=\beta+f^{\prime}(z) \eta_{\xi}+O\left(e^{-(1+\delta) \sqrt{f^{\prime}(z)} d_{\xi} / \epsilon}\right) .
$$


If we integrate this equation, then

$$
\overline{f^{\prime}\left(w_{\xi}\right)}=\beta+f^{\prime}(z) \eta_{\xi}+O\left(e^{-(1+\delta) \sqrt{f^{\prime}(z)} d_{\xi} / \epsilon}\right) .
$$

Therefore

$$
S\left(w_{\xi}\right)=-\epsilon^{2} \Delta w_{\xi}+f\left(w_{\xi}\right)-\overline{f\left(w_{\xi}\right)}=O\left(e^{-(1+\delta) \sqrt{f^{\prime}(z)} d_{\xi} / \epsilon}\right),
$$

and Lemma 5.4 is proved.

\section{Appendix D. Proof of Lemma 5.5}

To prove Lemma 5.5 , it suffices to prove the estimate. The one-to-one property follows immediately, and the onto property follows from the Fredholm alternative.

To simplify notation we omit subscript $\xi$ in quantities like $L_{\xi}, b_{1, \xi}$ and $b_{2, \xi}$. We prove the lemma by contradiction. Suppose that there exists $\phi$ such that $\|\phi\|_{W^{2,2}\left(\Omega_{\xi}\right)}=1$ and $\|\pi \circ L(\phi)\|_{L^{2}\left(\Omega_{\xi}\right)}=$ $o\left(\epsilon^{4 / 3}\right)$. Denote $\pi \circ L(\phi)$ by $q$ and $\overline{f^{\prime}(w) \phi}$ by $c_{0}$. Then we write the equation $\pi_{\xi} \circ L_{\xi}(\phi)=q$ as

$$
-\Delta \phi+f^{\prime}(w) \phi=c_{0}+c_{1} b_{1}+c_{2} b_{2}+q, \quad \bar{\phi}=0, \phi \perp b_{1}, \phi \perp b_{2},
$$

where $c_{1}$ and $c_{2}$, like $c_{0}$, are unknown constants.

We first consider a region in $\Omega$ that is far away from the droplet. Recall $\Omega_{\sigma}$ given in (5.1) and the small positive number $\sigma$ given in $(5.2)$. For any $\xi \in \Omega_{\sigma}, B_{5 \sigma}(\xi) \subset \Omega$. The functions $b_{1}$ and $b_{2}$ are supported in $B_{\sigma}(\xi)$. After rescaling, $B_{5 \sigma}(\xi)$ becomes $B_{5 \sigma / \epsilon}$ whose radius is $5 \sigma / \epsilon$ and center is the origin. In the region $\Omega_{\xi} \backslash B_{\sigma / \epsilon}$, we note that $\phi-c_{0} / f^{\prime}(z)$ satisfies the equation

$$
-\Delta\left(\phi-\frac{c_{0}}{f^{\prime}(z)}\right)+f^{\prime}(w)\left(\phi-\frac{c_{0}}{f^{\prime}(z)}\right)=q+O\left(e^{-C / \epsilon}\right)
$$

and the Neumann boundary condition on $\partial \Omega_{\xi}$. Let $\kappa$ be a smooth cut-off function so that $\kappa=1$ in $\Omega \backslash B_{2 \sigma}$ and $\kappa=0$ in $B_{\sigma}$. Then it is easy to see that

$$
\left\|-\Delta\left[\left(\phi-\frac{c_{0}}{f^{\prime}(z)}\right) \kappa\right]+f^{\prime}(w)\left[\left(\phi-\frac{c_{0}}{f^{\prime}(z)}\right) \kappa\right]\right\|_{L^{2}\left(\Omega_{\xi} \backslash B_{\sigma / \epsilon}\right)}=O(\epsilon) .
$$

By elliptic regularity theory,

$$
\left\|\phi-\frac{c_{0}}{f^{\prime}(z)}\right\|_{W^{2,2}\left(\Omega_{\xi} \backslash B_{2 \sigma / \epsilon}\right)}=O(\epsilon) .
$$

Next we consider $\phi$ in $B_{4 \sigma / \epsilon}$. Let $\psi$ be the solution of

$$
-\Delta \psi+f^{\prime}(z) \psi=0 \quad \text { in } B_{4 \sigma / \epsilon}, \quad \partial_{\nu} \psi=\partial_{\nu} \phi \quad \text { on } \partial B_{4 \sigma / \epsilon} .
$$

Define $\varphi=\phi-\psi-\alpha$ where $\alpha$ is the average of $\phi-\psi$ over $B_{4 \sigma / \epsilon}$ :

$$
\alpha=\frac{1}{\left|B_{4 \sigma / \epsilon}\right|} \int_{B_{4 \sigma / \epsilon}}(\phi-\psi) \mathrm{d} y .
$$


Note that $\varphi$ satisfies the Neumann boundary condition on $\partial B_{4 \sigma / \epsilon}$ and has zero average. The equation for $\varphi$ is

$$
-\Delta \varphi+f^{\prime}(w) \varphi=c_{0}+c_{1} b_{1}+c_{2} b_{2}+q+\left(f^{\prime}(z)-f^{\prime}(w)\right) \psi-\alpha f^{\prime}(w) .
$$

Since $\left(f^{\prime}(z)-f^{\prime}(w)\right) \psi=O\left(e^{-C / \epsilon}\right)$ for some $C>0$ independent of $\epsilon$, we write

$$
L_{B}(\varphi)=c_{1} b_{1}+c_{2} b_{2}+q-\operatorname{Av}(q)-\alpha\left(f^{\prime}(w)-\operatorname{Av}\left(f^{\prime}(w)\right)\right)+O\left(e^{-C / \epsilon}\right)
$$

where we define the linear operator $L_{B}$ in $B_{4 \sigma / \epsilon}$ by

$$
L_{B}(\varphi)=-\Delta \varphi+f^{\prime}(w) \varphi-\operatorname{Av}\left(f^{\prime}(w) \varphi\right),
$$

in which $\operatorname{Av}(\ldots)$ is the average of a function over $B_{4 \sigma / \epsilon}$.

We now use the results obtained in Sections 3 and 4. However, there the radius of the disc is 1, and here, before rescaling, the radius is $4 \sigma$. We could redo the two sections with the more general radius. But for simplicity we will just assume without the loss of generality that $4 \sigma=1$. Using complex notation we organize the eigenpairs by modes, i.e. $\lambda_{j l}$ and $e_{j l}$ where $j=0, \pm 1, \pm 2, \ldots$. and $l=1,2,3, \ldots$ Here $\lambda_{j l}=\lambda_{-j, l}$. Each $e_{j l}$ is normalized and takes the form

$$
e_{j l}=e^{2 \pi j \theta} \zeta_{j l}(r)
$$

$\lambda_{0,1}$ is the eigenvalue discussed in Theorem 4.1 and $\lambda_{ \pm j, 1}, j=1,2, \ldots$, are the critical eigenvalues discussed in Theorem 4.2. Up to translation, normalization and an exponentially small error caused by truncation, $e_{-1,1}$ is $b_{1}-i b_{2}$ and $e_{11}$ is $b_{1}+i b_{2}$. Decompose $\varphi$ so that

$$
\varphi=\sum_{j=-\infty}^{\infty} \sum_{l=1}^{\infty} d_{j l} e_{j l}
$$

where we let

$$
\begin{gathered}
\varphi^{\perp}=\sum_{l=2}^{\infty} d_{0 l} e_{0 l}, \quad \tilde{\varphi}=\sum_{l=2}^{\infty}\left(d_{1 l} e_{1 l}+d_{-1, l} e_{-1, l}\right)+\sum_{|j|=2}^{\infty} \sum_{l=1}^{\infty} d_{j l} e_{j l}, \\
d_{0}=d_{01}, \quad d_{ \pm 1}=d_{ \pm 1,1}, \quad e_{0}=e_{01}, \quad e_{ \pm 1}=e_{ \pm 1,1}
\end{gathered}
$$

so that

$$
\varphi=d_{0} e_{0}+d_{1} e_{1}+d_{-1} e_{-1}+\varphi^{\perp}+\tilde{\varphi} .
$$

One remark is in order. The linear operator $L_{B}$ here differs from the linear operator in Section 4 in that $L_{B}$ is linearization around $w$ while in Section 4 the linearization is around a solution. However, the difference between the two functions is exponentially small. Exponentially small quantities are negligible in this proof. Hence the $e_{j}$ 's are approximate eigenfunctions of $L_{B}$ :

$$
L_{B}\left(e_{j}\right)=\lambda_{j} e_{j}+O\left(e^{-C / \epsilon}\right), \quad j=0, \pm 1,
$$

where we have set $\lambda_{0}=\lambda_{01}$ and $\lambda_{1}=\lambda_{11}=\lambda_{-1}=\lambda_{-1,1}$. Theorems 4.1 and 4.2 show that there exists $c>0$ independent of $\epsilon$ so that

$$
\left\langle L_{B}\left(\varphi^{\perp}\right), \varphi^{\perp}\right\rangle \geqslant c\left\|\varphi^{\perp}\right\|_{L^{2}\left(B_{4 \sigma / \epsilon}\right)}^{2}
$$


and

$$
\left\langle L_{B}(\tilde{\varphi}), \tilde{\varphi}\right\rangle \geqslant c \epsilon^{4 / 3}\|\tilde{\varphi}\|_{L^{2}\left(B_{4 \sigma / \epsilon}\right)}^{2} .
$$

We claim that $d_{ \pm 1}$ are exponentially small. We have used $\langle$,$\rangle to denote the inner product in$ $L^{2}\left(B_{4 \sigma / \epsilon}\right)$. Note that $\phi \perp b_{1}, b_{2}$ in $L^{2}\left(\Omega_{\xi}\right)$ implies that $\left\langle\varphi+\psi+\alpha, e_{ \pm 1}\right\rangle=O\left(e^{-C / \epsilon}\right)$. It follows that $\left\langle\varphi, e_{ \pm 1}\right\rangle=O\left(e^{-C / \epsilon}\right)$. This implies that

$$
d_{ \pm 1}=O\left(e^{-C / \epsilon}\right) .
$$

In (D.4), $\tilde{\varphi}$ is also easy to analyze. We write the equation (D.3) as

$$
\begin{aligned}
\lambda_{0} d_{0} e_{0}+\lambda_{1} d_{1} e_{1}+\lambda_{1} d_{-1} e_{-1} & +L_{B}\left(\varphi^{\perp}\right)+L_{B}(\tilde{\varphi}) \\
& =c_{1} b_{1}+c_{2} b_{2}+q-\operatorname{Av}(q)-\alpha\left(f^{\prime}(w)-\operatorname{Av}\left(f^{\prime}(w)\right)\right) .
\end{aligned}
$$

Multiply $(\mathrm{D} .8)$ by $\tilde{\varphi}$ and integrate to obtain

$$
\left\langle L_{B}(\tilde{\varphi}), \tilde{\varphi}\right\rangle=\langle q, \tilde{\varphi}\rangle .
$$

Note that $\left\langle f^{\prime}(w), \tilde{\varphi}\right\rangle=0$ since $f^{\prime}(w)$ is radial and $\tilde{\varphi}$ is perpendicular to radial functions. Hence by (D.6),

$$
\|\tilde{\varphi}\|_{L^{2}\left(B_{4 \sigma / \epsilon}\right)}=o(1)
$$

The analysis of $d_{0}$ is trickier. It has to be done together with the estimation of $\varphi^{\perp}$. Multiply D.8 by $\varphi^{\perp}$ and integrate to find that

$$
\left\langle L_{B}\left(\varphi^{\perp}\right), \varphi^{\perp}\right\rangle=\left\langle q, \varphi^{\perp}\right\rangle-\alpha\left\langle f^{\prime}(w)-f^{\prime}(z), \varphi^{\perp}\right\rangle .
$$

Using (D.5) and the fact that

$$
\left\|f^{\prime}(w)-f^{\prime}(z)\right\|_{L^{2}\left(B_{4 \sigma / \epsilon}\right)}=O\left(\epsilon^{-1 / 3}\right)
$$

we find that

$$
\left\|\varphi^{\perp}\right\|_{L^{2}\left(B_{4 \sigma / \epsilon}\right)}=o\left(\epsilon^{4 / 3}\right)+\alpha O\left(\epsilon^{-1 / 3}\right) .
$$

Multiply (D.8) by $e_{0}$ and integrate to find (since $\left\|e_{0}\right\|_{L^{2}\left(B_{4 \sigma / \epsilon}\right)}=1$ )

$$
\lambda_{0} d_{0}=\left\langle q, e_{0}\right\rangle-\alpha\left\langle f^{\prime}(w), e_{0}\right\rangle
$$

Since $\lambda_{0} \sim \epsilon^{4 / 3}$ and $\|q\|_{L^{2}\left(B_{4 \sigma / \epsilon}\right)}=o\left(\epsilon^{4 / 3}\right)$, we deduce

$$
d_{0}=o(1)-\frac{\left\langle f^{\prime}(w), e_{0}\right\rangle \alpha}{\lambda_{0}} .
$$


Calculations show that

$$
\begin{aligned}
c_{0} & =\frac{\epsilon^{2}}{|\Omega|} \int_{\Omega_{\xi}} f^{\prime}(w) \phi \mathrm{d} y=\frac{\epsilon^{2}}{|\Omega|} \int_{\Omega_{\xi}}\left(f^{\prime}(w)-f^{\prime}(z)\right) \phi \mathrm{d} y \\
& =\frac{\epsilon^{2}}{|\Omega|} \int_{B_{4 \sigma / \epsilon}}\left(f^{\prime}(w)-f^{\prime}(z)\right) \phi \mathrm{d} y+O\left(e^{-C / \epsilon}\right) \\
& =\frac{\epsilon^{2}}{|\Omega|} \int_{B_{4 \sigma / \epsilon}}\left(f^{\prime}(w)-f^{\prime}(z)\right)\left(d_{0} e_{0}+d_{1} e_{1}+d_{-1} e_{-1}+\varphi^{\perp}+\tilde{\varphi}+\alpha\right) \mathrm{d} y+O\left(e^{-C / \epsilon}\right) \\
& =\frac{\epsilon^{2}}{|\Omega|} \int_{B_{4 \sigma / \epsilon}}\left(f^{\prime}(w)-f^{\prime}(z)\right)\left(d_{0} e_{0}+\varphi^{\perp}+\alpha\right) \mathrm{d} y+O\left(e^{-C / \epsilon}\right) \\
& =\frac{\epsilon^{2}\left\langle f^{\prime}(w), e_{0}\right\rangle d_{0}}{|\Omega|}+\left\|f^{\prime}(w)-f^{\prime}(z)\right\|_{L^{2}\left(B_{4 \sigma / \epsilon}\right)}\left\|\varphi^{\perp}\right\|_{L^{2}\left(B_{4 \sigma / \epsilon}\right)} O\left(\epsilon^{2}\right)+\alpha O\left(\epsilon^{4 / 3}\right) \\
& =\frac{\epsilon^{2}\left\langle f^{\prime}(w), e_{0}\right\rangle d_{0}}{|\Omega|}+\alpha O\left(\epsilon^{4 / 3}\right)+o\left(\epsilon^{3}\right)
\end{aligned}
$$

where the last line follows from $(\mathrm{D} .10)$. Thus we have the important fact that

$$
c_{0}=\frac{\epsilon^{2}\left\langle f^{\prime}(w), e_{0}\right\rangle d_{0}}{|\Omega|}+\alpha O\left(\epsilon^{4 / 3}\right)+o\left(\epsilon^{3}\right) .
$$

The calculations in Appendix A between (A.2) and (A.3) show that

$$
\left\langle f^{\prime}(w), e_{0}\right\rangle=\sqrt{\frac{2 \rho_{0} \pi}{\tau}} f^{\prime}(0) \epsilon^{-1 / 3}+o\left(\epsilon^{-1 / 3}\right)
$$

since $e_{0}$ is essentially the scaled and normalized version of $p$ there. Plugging (D.11) into (D.12) and using D.13 we find

$$
c_{0}=-\frac{\left\langle f^{\prime}(w), e_{0}\right\rangle^{2} \epsilon^{2} \alpha}{\lambda_{0}|\Omega|}+\alpha O\left(\epsilon^{4 / 3}\right)+o\left(\epsilon^{5 / 3}\right) .
$$

We consider $\phi$ in the matching region $B_{3 \sigma / \epsilon} \backslash B_{2 \sigma / \epsilon}$. Since the $L^{2}$ norm of $\phi-c_{0} / f^{\prime}(z)$ is of order $O(\epsilon)$ in this region by (D.2), and $\psi$ in $\phi=\varphi+\psi+\alpha$ is exponentially small, we find that

$$
\begin{aligned}
\| d_{0} e_{0}+\tilde{\varphi} & +\alpha-\frac{c_{0}}{f^{\prime}(z)} \|_{L^{2}\left(B_{3 \sigma / \epsilon} \backslash B_{2 \sigma / \epsilon}\right)} \\
& \leqslant\left\|\phi-\frac{c_{0}}{f^{\prime}(z)}\right\|_{L^{2}\left(B_{3 \sigma / \epsilon} \backslash B_{2 \sigma / \epsilon}\right)}+\left\|\varphi^{\perp}\right\|_{L^{2}\left(B_{3 \sigma / \epsilon} \backslash B_{2 \sigma / \epsilon}\right)}+\left|d_{1}\right|+\left|d_{-1}\right|+O\left(e^{-C / \epsilon}\right) \\
& =O(\epsilon)+o\left(\epsilon^{4 / 3}\right)+\alpha O\left(\epsilon^{-1 / 3}\right)=O(\epsilon)+\alpha O\left(\epsilon^{-1 / 3}\right)
\end{aligned}
$$

by (D.10). Because $d_{0} e_{0}+\alpha-c_{0} / f^{\prime}(z)$ is still orthogonal to $\tilde{\varphi}$ in this region, we write

$$
\begin{aligned}
\left\|d_{0} e_{0}+\tilde{\varphi}+\alpha-\frac{c_{0}}{f^{\prime}(z)}\right\|_{L^{2}\left(B_{3 \sigma / \epsilon} \backslash B_{2 \sigma / \epsilon}\right)}^{2} & =\|\tilde{\varphi}\|_{L^{2}\left(B_{3 \sigma / \epsilon} \backslash B_{2 \sigma / \epsilon}\right)}^{2}+\left\|d_{0} e_{0}+\alpha-\frac{c_{0}}{f^{\prime}(z)}\right\|_{L^{2}\left(B_{3 \sigma / \epsilon} \backslash B_{2 \sigma / \epsilon}\right)}^{2} \\
& \geqslant\left\|d_{0} e_{0}+\alpha-\frac{c_{0}}{f^{\prime}(z)}\right\|_{L^{2}\left(B_{3 \sigma / \epsilon} \backslash B_{2 \sigma / \epsilon}\right)}^{2} \cdot
\end{aligned}
$$


Therefore we conclude that

$$
\left\|d_{0} e_{0}+\alpha-\frac{c_{0}}{f^{\prime}(z)}\right\|_{L^{2}\left(B_{3 \sigma / \epsilon} \backslash B_{2 \sigma / \epsilon}\right)}=\alpha O\left(\epsilon^{-1 / 3}\right)+O(\epsilon) .
$$

In this matching region, uniformly in $y$, as the scaled and normalized version of $p, e_{0}$ satisfies the estimate

It follows from (D.15) that

$$
e_{0}(y)=\sqrt{\frac{2 \rho_{0}}{\pi \tau}} \epsilon^{5 / 3}+o\left(\epsilon^{5 / 3}\right) .
$$

$$
\begin{gathered}
\left\|d_{0} \sqrt{\frac{2 \rho_{0}}{\pi \tau}} \epsilon^{5 / 3}+\alpha-\frac{c_{0}}{f^{\prime}(z)}\right\|_{L^{2}\left(B_{3 \sigma / \epsilon \backslash B_{2 \sigma / \epsilon}}\right)} \\
\leqslant\left\|d_{0} e_{0}+\alpha-\frac{c_{0}}{f^{\prime}(z)}\right\|_{L^{2}\left(B_{3 \sigma / \epsilon} \backslash B_{2 \sigma / \epsilon}\right)}+\left\|d_{0} o\left(\epsilon^{5 / 3}\right)\right\|_{L^{2}\left(B_{3 \sigma / \epsilon} \backslash B_{2 \sigma / \epsilon}\right)} \\
\leqslant a O\left(\epsilon^{-1 / 3}\right)+d_{0} o\left(\epsilon^{2 / 3}\right)+O(\epsilon),
\end{gathered}
$$

which implies that

$$
d_{0} \sqrt{\frac{2 \rho_{0}}{\pi \tau}} \epsilon^{5 / 3}+\alpha-\frac{c_{0}}{f^{\prime}(z)}=\alpha O\left(\epsilon^{2 / 3}\right)+d_{0} o\left(\epsilon^{5 / 3}\right)+O\left(\epsilon^{2}\right)
$$

Upon substitution of (D.11) and (D.14) we deduce that

$$
-\frac{\left\langle f^{\prime}(w), e_{0}\right\rangle \alpha}{\lambda_{0}} \sqrt{\frac{2 \rho_{0}}{\pi \tau}} \epsilon^{5 / 3}+\alpha+\frac{\left\langle f^{\prime}(w), e_{0}\right\rangle^{2} \epsilon^{2} \alpha}{\lambda_{0}|\Omega| f^{\prime}(z)}=\alpha O\left(\epsilon^{2 / 3}\right)+o\left(\epsilon^{5 / 3}\right) .
$$

This shows, from Theorem 4.1 and (D.13), that

$$
\alpha\left[1-\frac{\frac{2 \rho_{0} f^{\prime}(0)}{\tau}\left(1-\frac{\pi}{|\Omega|}\right)}{\frac{2 \rho_{0} f^{\prime}(0)}{\tau}-\frac{1}{\rho_{0}^{2}}}+o(1)\right]=o\left(\epsilon^{5 / 3}\right) .
$$

Note that the big fraction is 1 precisely when $\rho_{0}^{3}=|\Omega| \tau /\left(2 \pi f^{\prime}(0)\right)$, which is attained at the critical mass. Under the assumption of Theorem 5.1. $\rho_{0}^{3} \neq|\Omega| \tau /\left(2 \pi f^{\prime}(0)\right)$ (see Lemma 5.3. Hence the fraction is not 1 and we conclude that

$$
\alpha=o\left(\epsilon^{5 / 3}\right) \quad \text { and hence } \quad d_{0}=o(1), c_{0}=o\left(\epsilon^{5 / 3}\right)
$$

by (D.11) and (D.14). Moreover, from (D.10) we have

$$
\left\|\varphi^{\perp}\right\|_{L^{2}\left(B_{4 \sigma / \epsilon}\right)}=o\left(\epsilon^{4 / 3}\right) .
$$

It follows from (D.7), $\overline{\mathrm{D} .9}, \overline{\mathrm{D} .16)}$ and $\left(\overline{\mathrm{D} .17)}\right.$ that $\|\varphi\|_{L^{2}\left(B_{4 \sigma / \epsilon}\right)}=o(1)$, and consequently $\|\phi\|_{L^{2}\left(B_{3 \sigma / \epsilon}\right)}=o(1)$. In the region $\Omega_{\xi} \backslash B_{2 \sigma / \epsilon}$ we have $\|\phi\|_{W^{2,2}\left(\Omega_{\xi} \backslash B_{2 \sigma / \epsilon}\right)}=o\left(\epsilon^{2 / 3}\right)$ by $(\mathrm{D} .2$ ) and $c_{0}=o\left(\epsilon^{5 / 3}\right)$. In the whole region $\Omega_{\xi}$ we have

$$
\|\phi\|_{L^{2}\left(\Omega_{\xi}\right)}=o(1) .
$$


Rewrite the equation for $\phi$ as

$$
-\Delta \phi+f^{\prime}(z) \phi=\left(f^{\prime}(z)-f^{\prime}(w)\right) \phi+c_{0}+c_{1} b_{1}+c_{2} b_{2}+q .
$$

The elliptic regularity theory asserts that

$$
\|\phi\|_{W^{2,2}\left(\Omega_{\xi}\right)} \leqslant C\left\|\left(f^{\prime}(z)-f^{\prime}(w)\right) \phi+c_{0}+c_{1} b_{1}+c_{2} b_{2}+q\right\|_{L^{2}\left(\Omega_{\xi}\right)}
$$

where $C$ is independent of $\epsilon$. The only quantities that remain to be estimated are $c_{1}$ and $c_{2}$. Multiply the equation $(\mathrm{D} .1)$ for $\phi$ by $b_{j}, j=1,2$, and integrate to find

$$
\lambda_{1} \int_{\Omega_{\xi}} \phi\left(b_{j}+O\left(e^{-C / \epsilon}\right)\right) \mathrm{d} y=c_{j}\left\|b_{j}\right\|_{L^{2}\left(\Omega_{\xi}\right)}^{2}+\int_{\Omega_{\xi}} q b_{j} \mathrm{~d} y .
$$

Hence, for $j=1,2$, since $\phi \perp b_{j}$ in $L^{2}\left(\Omega_{\xi}\right)$,

$$
c_{j}=\frac{O\left(e^{-C / \epsilon}\right)-\int_{\Omega_{\xi}} q b_{j} \mathrm{~d} y}{\left\|b_{j}\right\|_{L^{2}\left(\Omega_{\xi}\right)}^{2}}=\frac{O\left(\|q\|_{L^{2}\left(\Omega_{\xi}\right)}\right)}{\left\|b_{j}\right\|_{L^{2}\left(\Omega_{\xi}\right)}}=\frac{o\left(\epsilon^{4 / 3}\right)}{\left\|b_{j}\right\|_{L^{2}\left(\Omega_{\xi}\right)}} .
$$

It follows from $(\mathrm{D} .18)$ that $\|\phi\|_{W^{2,2}\left(\Omega_{\xi}\right)}=o(1)$ and we have a contradiction to the assumption that $\|\phi\|_{W^{2,2}\left(\Omega_{\xi}\right)}=1$.

\section{Appendix E. Proof of Lemma 6.4}

The constant $\eta_{\xi}$ in the definition of $w_{\xi}$ satisfies

$$
\eta_{\xi}=-\frac{1}{f^{\prime}(z)|\Omega|} \int_{\mathbb{R}^{2} \backslash \Omega} \sum_{k=1}^{K} h\left(\tilde{v}_{k}\right) d x .
$$

It follows as in Appendix $\mathrm{C}$ that

$$
\left|\eta_{\xi}\right| \leqslant-C \epsilon^{2} \int_{\partial \Omega} \sum_{k=1}^{K} \frac{\partial \tilde{v}_{k}}{\partial v} \tilde{v}_{k} \mathrm{~d} s+O\left(e^{-(2+\delta) \sqrt{f^{\prime}(z)} \varphi(\xi) / \epsilon}\right)
$$

and

$$
\eta_{\xi}=O\left(e^{-2 \sqrt{f^{\prime}(z)} \varphi(\xi) / \epsilon}\right)
$$

The functional $I\left(w_{\xi}\right)$ can be written as

$$
I\left(w_{\xi}\right)=\tilde{I}\left(\tilde{w}_{\xi}\right)+|\Omega|(F(z)+f(z)(m-z))+O\left(\epsilon^{2 / 3} \eta_{\xi}\right)
$$

where the second term on the right side is independent of $\xi$ and

$$
\tilde{I}\left(\tilde{w}_{\xi}\right)=\int_{\Omega}\left[\frac{\epsilon^{2}\left|\nabla \tilde{w}_{\xi}\right|^{2}}{2}+\frac{f^{\prime}(z)}{2} \tilde{w}_{\xi}^{2}+H\left(\tilde{w}_{\xi}\right)\right] \mathrm{d} x .
$$

To estimate the first term we note an important but trivial fact

$$
C_{1} \tilde{v}^{2} \leqslant-h(\tilde{v}) \leqslant C_{2} \tilde{v}^{2}, \quad C_{1}, C_{2}>0,
$$


because of the assumption $F^{\prime \prime \prime}(0)<0$. This implies that

$$
\begin{aligned}
\int_{\Omega}\left(-h\left(\tilde{v}_{i}\right) \tilde{v}_{j}\right) & \leqslant C \int_{\Omega} \tilde{v}_{i}^{2} \tilde{v}_{j} \leqslant C \epsilon^{a \epsilon^{-2 / 3}} \int_{\Omega} e^{-2 \sqrt{f^{\prime}(z)}\left|x-\xi^{i}\right| / \epsilon} e^{-\sqrt{f^{\prime}(z)\left|x-\xi^{j}\right| / \epsilon}} \\
& \leqslant C \epsilon^{a \epsilon^{-2 / 3}} e^{-\sqrt{f^{\prime}(z)\left|\xi^{i}-\xi^{j}\right| / \epsilon}} .
\end{aligned}
$$

Similarly we have a lower bound and

$$
-C_{1} \epsilon^{a_{1} \epsilon^{-2 / 3}} e^{-\sqrt{f^{\prime}(z)}\left|\xi^{i}-\xi^{j}\right| / \epsilon} \leqslant \int_{\Omega} h\left(\tilde{v}_{i}\right) \tilde{v}_{j} \leqslant-C_{0} \epsilon^{-a_{0} \epsilon^{-2 / 3}} e^{-\sqrt{f^{\prime}(z)}\left|\xi^{i}-\xi^{j}\right| / \epsilon} .
$$

Let $\tilde{w}_{k}=\tilde{v}_{k}+g_{k}$. We compare $\tilde{I}\left(\tilde{w}_{\xi}\right)$ with $\sum_{k=1}^{K} \tilde{I}\left(\tilde{w}_{k}\right)$ :

$$
\begin{aligned}
\tilde{I}\left(\sum_{k=1}^{K} \tilde{w}_{k}\right)= & \sum_{k=1}^{K} \tilde{I}\left(\tilde{w}_{k}\right)+\frac{1}{2} \sum_{i \neq j} \int_{\Omega}\left[\epsilon^{2} \nabla \tilde{w}_{i} \nabla \tilde{w}_{j}+f^{\prime}(z) \tilde{w}_{i} \tilde{w}_{j}\right] \mathrm{d} x \\
& +\int_{\Omega}\left[H\left(\sum_{k=1}^{K} \tilde{w}_{k}\right)-\sum_{k=1}^{K} H\left(\tilde{w}_{k}\right)\right] \mathrm{d} x .
\end{aligned}
$$

Using the equation for $\tilde{w}_{i}$, we see that

$$
\int_{\Omega}\left[\epsilon^{2} \nabla \tilde{w}_{i} \nabla \tilde{w}_{j}+f^{\prime}(z) \tilde{w}_{i} \tilde{w}_{j}\right]=-\int_{\Omega} h\left(\tilde{v}_{i}\right) \tilde{w}_{j} .
$$

Next, since

$$
\left|h\left(\tilde{w}_{i}\right)-h\left(\tilde{v}_{i}\right)\right|\left|\tilde{w}_{j}\right| \leqslant C\left(\left|\tilde{w}_{i}\right|+\left|\tilde{v}_{i}\right|\right)\left|g_{i}\right|\left|\tilde{w}_{j}\right|
$$

we obtain

$$
\int_{\Omega}\left|h\left(\tilde{w}_{i}\right)-h\left(\tilde{v}_{i}\right)\right| \tilde{w}_{j}=O\left(e^{-(2+\delta) \sqrt{f^{\prime}(z)} \varphi\left(\xi_{1}, \ldots, \xi_{K}\right) / \epsilon}\right) .
$$

It follows that

$$
\begin{aligned}
\int_{\Omega}\left[H\left(\sum_{k=1}^{K} \tilde{w}_{k}\right)-\sum_{k=1}^{K} H\left(\tilde{w}_{k}\right)\right] & =\sum_{i \neq j} \int_{\Omega} h\left(\tilde{w}_{i}\right) \tilde{w}_{j}+O\left(\sum_{i \neq j} \int_{\Omega}\left|\tilde{w}_{i}\right|^{2}\left|\tilde{w}_{j}\right|^{2}\right) \\
& =\sum_{i \neq j} \int_{\Omega} h\left(\tilde{v}_{i}\right) \tilde{w}_{j}+O\left(e^{-(2+\delta) \sqrt{f^{\prime}(z)} \varphi\left(\xi_{1}, \ldots, \xi_{K}\right) / \epsilon}\right) .
\end{aligned}
$$

Therefore

$$
\begin{aligned}
I\left(\sum_{k=1}^{K} \tilde{w}_{k}\right)= & \sum_{k=1}^{K} \tilde{I}\left[\tilde{w}_{k}\right]+\frac{1}{2} \sum_{i \neq j} \int_{\Omega} h\left(\tilde{v}_{i}\right) \tilde{w}_{j} \mathrm{~d} x+|\Omega|(F(z)+f(z)(m-K z)) \\
& +O\left(\epsilon^{2 / 3}|\eta \xi|+e^{-(2+\delta) \sqrt{f^{\prime}(z)} \varphi\left(\xi^{1}, \ldots, \xi^{K}\right) / \epsilon}\right) .
\end{aligned}
$$

By Lemma C.1.2), we have

$$
-\int_{\Omega} h\left(\tilde{v}_{i}\right)\left(\tilde{v}_{j}+g_{j}\right) \geqslant C \epsilon^{a \epsilon^{-2 / 3}} e^{-\sqrt{f^{\prime}(z)}\left|\xi^{i}-\xi^{j}\right| / \epsilon}-C e^{-(1+\delta) \sqrt{f^{\prime}(z)} d_{\xi^{j}} / \epsilon}\left(-\int_{\Omega} h\left(\tilde{v}_{i}\right) \hat{g}\right)
$$


where

$$
-\int_{\Omega} h\left(\tilde{v}_{i}\right) \hat{g}=\epsilon^{2} \int_{\partial \Omega}\left(\tilde{v}_{i}-\hat{g} \frac{\partial \tilde{v}_{i}}{\partial v}\right)=O\left(e^{a \epsilon^{-2 / 3}} e^{-\sqrt{f^{\prime}(z)} d_{\xi^{i}} / \epsilon}\right) .
$$

Hence by (E.1),

$$
-\int_{\Omega} h\left(\tilde{v}_{i}\right) \tilde{w}_{j} \geqslant C e^{a \epsilon^{-2 / 3}} e^{-\sqrt{f^{\prime}(z)}\left|\xi_{1}-\xi_{2}\right| / \epsilon}-O\left(e^{-(2+\delta) \sqrt{f^{\prime}(z)} \varphi\left(\xi_{1}, \ldots, \xi_{K}\right) / \epsilon}\right) .
$$

By Lemma C.1 (1) and (E.1), we also have

$$
-\int_{\Omega} h\left(\tilde{v}_{i}\right) g_{j} \leqslant e^{-\sqrt{f^{\prime}(z)} d_{\xi^{j}} / \epsilon} \int_{\Omega}\left(-h\left(\tilde{v}_{i}\right)\right) \hat{g} \leqslant C \epsilon^{a \epsilon^{-2 / 3}} e^{-\sqrt{f^{\prime}(z)}\left(d_{\xi^{i}}+d_{\xi^{j}}\right) / \epsilon} .
$$

Hence

$$
-\int_{\Omega} h\left(\tilde{v}_{i}\right) \tilde{w}_{j} \leqslant C e^{a \epsilon^{-2 / 3}}\left[e^{-\sqrt{f^{\prime}(z)}\left|\xi^{i}-\xi^{j}\right| / \epsilon}+e^{-\sqrt{f^{\prime}(z)}\left(d_{\xi^{i}}+d_{\xi^{j}}\right) / \epsilon}\right] .
$$

In Appendix $\mathrm{C}$ we have learned that $\tilde{I}\left(\tilde{w}_{k}\right)$ is estimated in (C.6), the most dominating term in (C.4). Combining it with $(E .2)$ and $($ E.3 $)$, we see that the exponential decay rates in these terms are given by $\left|\xi^{i}-\xi^{j}\right|, d_{\xi^{i}}+d_{\xi^{j}}$, and $2 d_{\xi^{k}}$. Therefore the slowest decay rate is $\varphi\left(\xi^{1}, \ldots, \xi^{K}\right)$. This proves Lemma6.4(2).

To prove Lemma 6.4(1), we note that

$$
\begin{aligned}
-\epsilon^{2} \Delta w_{\xi}+f\left(w_{\xi}\right) & =-\epsilon^{2} \sum_{k=1}^{K} \Delta \tilde{w}_{k}+f\left(\sum_{k=1}^{K} \tilde{w}_{k}+z+\eta_{\xi}\right) \\
& =-f^{\prime}(z) \sum_{k=1}^{K} \tilde{w}_{k}-\sum_{k=1}^{K} h\left(\tilde{v}_{k}\right)+f(z)+f^{\prime}(z)\left(\sum_{k=1}^{K} \tilde{w}_{k}+\eta_{\xi}\right)+h\left(\sum_{k=1}^{K} \tilde{w}_{\xi}+\eta_{\xi}\right) \\
& =f(z)+f^{\prime}(z) \eta_{\xi}+h\left(\sum_{k=1}^{K} \tilde{w}_{\xi}+\eta_{\xi}\right)-\sum_{k=1}^{K} h\left(\tilde{v}_{k}\right) .
\end{aligned}
$$

We only need to focus on, as in Appendix C,

$$
\begin{aligned}
h\left(\sum_{k=1}^{K} \tilde{w}_{k}+\eta_{\xi}\right)-\sum_{k=1}^{K} h\left(\tilde{v}_{k}\right) & =h\left(\sum_{k=1}^{K} \tilde{w}_{k}\right)-\sum_{k=1}^{K} h\left(\tilde{v}_{k}\right)+O\left(e^{-2 \sqrt{f^{\prime}(z)} \varphi(\xi) / \epsilon}\right) \\
= & h\left(\sum_{k=1}^{K} \tilde{w}_{k}\right)-\sum_{k=1}^{K} h\left(\tilde{w}_{k}\right)+\sum_{k=1}^{K}\left[h\left(\tilde{w}_{k}\right)-h\left(\tilde{v}_{k}\right)\right]+O\left(e^{-2 \sqrt{f^{\prime}(z)} \varphi(\xi) / \epsilon}\right) \\
= & \sum_{i \neq j} O\left(\left|\tilde{w}_{i} \| \tilde{w}_{j}\right|\right)+O\left(e^{-(1+\delta) \sqrt{f^{\prime}(z)} \varphi(\xi) / \epsilon}\right)=O\left(e^{-(1+\delta) \sqrt{f^{\prime}(z)} \varphi(\xi) / \epsilon}\right) .
\end{aligned}
$$

This completes the proof.

\section{Appendix F. Proof of Lemma 6.5}

It suffices to prove the estimate. Assume on the contrary $\|\phi\|_{W^{2,2}\left(\Omega_{\xi}\right)}=1$ and $\left\|\pi_{\xi} \circ L_{\xi}(\phi)\right\|_{L^{2}\left(\Omega_{\xi}\right)}=$ $o\left(\epsilon^{4 / 3}\right)$. 
Let $\sigma>0$ be the small number given in 6.1 , which is independent of $\epsilon$, so that the $B_{5 \sigma}\left(\xi^{k}\right)$ 's are mutually disjoint and contained in $\Omega$. Denote $\overline{f^{\prime}(w) \phi}$ by $c_{0}$. In $\Omega_{\xi} \backslash \bigcup_{k} B_{2 \sigma / \epsilon}\left(\xi^{k}\right)$ we have, as in Appendix D,

$$
\left\|\phi-\frac{c_{0}}{f^{\prime}(z)}\right\|_{W^{2,2}\left(\Omega_{\xi} \backslash \bigcup_{k} B_{2 \sigma / \epsilon}\left(\xi^{k}\right)\right)}=O(\epsilon) .
$$

Next we consider $\phi$ in each $B_{4 \sigma / \epsilon}\left(\xi^{k}\right)$. Let $\psi^{k}$ be the solution of

$$
-\Delta \psi^{k}+f^{\prime}(z) \psi^{k}=0 \quad \text { in } B_{4 \sigma / \epsilon}\left(\xi^{k}\right), \quad \partial_{\nu} \psi^{k}=\partial_{\nu} \phi \quad \text { on } \partial B_{4 \sigma / \epsilon}\left(\xi^{k}\right) .
$$

Define $\varphi^{k}$ to be $\phi-\psi^{k}-\alpha^{k}$ where $\alpha^{k}$ is the average of $\phi-\psi^{k}$ in the ball:

$$
\alpha^{k}=\frac{1}{\left|B_{4 \sigma / \epsilon}\left(\xi^{k}\right)\right|} \int_{B_{4 \sigma / \epsilon}\left(\xi^{k}\right)}\left(\phi-\psi^{k}\right) \mathrm{d} y .
$$

We follow the same argument as in the proof of Lemma 5.5 and arrive at

$$
\left(1-\lambda_{0}^{-1} \tilde{e}_{0}\left\langle f^{\prime}(w), e_{0}\right\rangle\right) \alpha^{k}+\sum_{k=1}^{K} \frac{\left\langle f^{\prime}(w), e_{0}\right\rangle^{2} \epsilon^{2}}{\lambda_{0}|\Omega| f^{\prime}(z)} \alpha^{k}=o\left(\epsilon^{5 / 3}\right), \quad k=1, \ldots, K .
$$

We sum these $K$ equations to deduce

$$
\left(\sum_{k=1}^{K} \alpha^{k}\right)\left[1-\frac{\frac{2 \rho_{0} f^{\prime}(0)}{\tau}\left(1-\frac{K \pi}{|\Omega|}\right)}{\frac{2 f^{\prime}(0) \rho_{0}}{\tau}-\frac{1}{\rho_{0}^{2}}}+o(1)\right]=o\left(\epsilon^{5 / 3}\right) .
$$

Note that the big fraction is 1 precisely when $\rho_{0}^{3}=|\Omega| \tau /\left(2 K \pi f^{\prime}(0)\right)$, which is attained at the critical mass. When the mass is larger, this fraction is not 1 and we conclude that

$$
\sum_{k=1}^{K} \alpha^{k}=o\left(\epsilon^{5 / 3}\right)
$$

We now return to $($ F.1) to find that

$$
\alpha^{k}=o\left(\epsilon^{5 / 3}\right)
$$

for each $k$, because

$$
1-\lambda_{0}^{-1} \tilde{e}_{0}\left\langle f^{\prime}(w), e_{0}\right\rangle=1-\frac{\frac{2 \rho_{0} f^{\prime}(0)}{\tau}}{\frac{2 f^{\prime}(0) \rho_{0}}{\tau}-\frac{1}{\rho_{0}^{2}}}+o(1),
$$

which does not tend to 0 as $\epsilon \rightarrow 0$. The rest of the proof is the same as in Appendix D.

\section{Acknowledgments}

Research of X. Ren was supported in part by NSF grant DMS-0509725.

Research of J. Wei was supported in part by an Earmarked Grant of RGC of Hong Kong (RGC Proj. 402304). 


\section{REFERENCES}

1. Alikakos, N., Bronsard, L., \& Fusco, G. Slow motion in the gradient theory of phase transitions via energy and spectrum. Calc. Var. Partial Differential Equations 6 (1998), 39-66. Zbl 0910.35146 MR 1488493

2. Alikakos, N., Chen, X., \& Fusco, G. Motion of a droplet by surface tension along the boundary. Calc. Var. Partial Differential Equations 11 (2000), 233-305. Zbl 0994.35052 MR 1797871

3. Alikakos, N., \& Fusco, G. Slow dynamics for the Cahn-Hilliard equation in higher space dimension, part I: Spectral estimates. Comm. Partial Differential Equations 19 (1994), 1397-1447. Z Zbl 0814.35042 MR 1294466

4. Alikakos, N., \& Fusco, G. Slow dynamics for the Cahn-Hilliard equation in higher space dimension: The motion of bubbles. Arch. Ration. Mech. Anal. 141 (1998), 1-61. Zbl 0906.35049 MR 1613496

5. Alikakos, N., Fusco, G., \& Karali, G. Motion of bubbles towards the boundary for the CahnHilliard equation. Eur. J. Appl. Math. 15 (2004), 103-124. Zbl 1065.35136 MR 2056331

6. BAtes, P. W., DANCER, E. N., \& SHI, J. Multi-spike stationary solutions of the Cahn-Hilliard equation in higher dimension and instability. Adv. Differential Equations 4 (1999), 1-69. Zbl pre01493588 MR 1667283

7. BAtEs, P. W., \& FifE, P. C. The dynamics of nucleation for the Cahn-Hilliard equation. SIAM J. Appl. Math. 53 (1993), 990-1008. Zbl 0788.35061 MR 1232163

8. BAtes, P. W., \& FusCO, G. Equilibria with many nuclei for the Cahn-Hilliard equation. J. Differential Equations 160 (2000), 283-356. Zbl 0990.35016 MR 1737000

9. Cahn, J. W., \& Hilliard, J. E. Free energy of a nonuniform system. I. Interfacial free energy. J. Chem. Phys. 28 (1958), 258-267.

10. Chen, X., \& KowalczyK, M. Existence of equilibria for the Cahn-Hilliard equation via local minimizers of the perimeter. Comm. Partial Differential Equations 21 (1996), 1207-1233. Zbl 0863.35009 MR 1399196

11. De Giorgi, E. Sulla convergenza di alcune successioni d'integrali del tipo dell'area. Rend. Mat. (6) 8 (1975), 277-294. Zbl 0316.35036 MR 0375037

12. GuI, C., \& WEI, J. Multiple interior peak solutions for some singular perturbation problems. J. Differential Equations 158 (1999), 1-27. Zbl 1061.35502 MR 1721719

13. Kohn, R., \& Sternberg, P. Local minimisers and singular perturbations. Proc. Roy. Soc. Edinburgh Sect. A 111 (1989), 69-84. Zbl 0676.49011 MR 0985990

14. ModicA, L. The gradient theory of phase transitions and the minimal interface criterion. Arch. Ration. Mech. Anal. 98 (1987), 123-142. Zbl 0616.76004 MR 0866718

15. ModicA, L., \& Mortola, S. Un esempio di $\Gamma^{-}$-convergenza. Boll. Un. Mat. Ital. B (5) 14 (1977), 285-299. Zbl 0356.49008 MR 0445362

16. REN, X., \& WEI, J. Stability of spot and ring solutions of the diblock copolymer equation. J. Math. Phys. 45 (2004), 4106-4133. Zbl 1064.82040 MR 2098120

17. Sternberg, P., \& Zeimer, W. Local minimisers of a three-phase partition problem with triple junctions. Proc. Roy. Soc. Edinburgh Sect. A 124 (1994), 1059-1073. Zbl 0843.49025 MR 1313189

18. WARD, M. Metastable bubble solutions for the Allen-Cahn equation with mass conservation. SIAM J. Appl. Math. 56 (1996), 1247-1279. Zbl 0870.35011 MR 1409118

19. Wei, J., \& Winter, M. On the stationary Cahn-Hilliard equation: Bubble solutions. SIAM. J. Math. Anal. 29 (1998), 1492-1518. Zbl 0918.35023 MR 1636688

20. WeI, J., \& Winter, M. Stationary solutions for the Cahn-Hilliard equation. Ann. Inst. H. Poincaré Anal. Non Linéaire 15 (1998), 459-492. Zbl 0910.35049 MR 1632937

21. WeI, J., \& Winter, M. Multi-interior-spike solutions for the Cahn-Hilliard equation with arbitrarily many peaks. Calc. Var. Partial Differential Equations 10 (2000), 249-289. Zbl 0952.35029 MR 1756329 